\title{
Addressing Data Resiliency for Staging Based Scientific Workflows
}

\author{
Shaohua Duan, Pradeep Subedi, Philip E. Davis, Manish Parashar \\ shaohua.duan@rutgers.edu,ps917@ored.rutgers.edu,philip.e.davis@rutgers.edu,parashar@ored.rutgers.edu \\ Rutgers Discovery Informatics Institute, Rutgers University \\ Piscataway, NJ, USA
}

\begin{abstract}
As applications move towards extreme scales, data-related challenges are becoming significant concerns, and in-situ workflows based on data staging and in-situ/in-transit data processing have been proposed to address these challenges. Increasing scale is also expected to result in an increase in the rate of silent data corruption errors, which will impact both the correctness and performance of applications. Furthermore, this impact is amplified in the case of in-situ workflows due to the dataflow between the component applications of the workflow. While existing research has explored silent error detection at the application level, silent error detection for workflows remains an open challenge. This paper addresses silent error detection for extreme scale in-situ workflows. The presented approach leverages idle computation resource in data staging to enable timely detection and recovery from silent data corruption, effectively reducing the propagation of corrupted data and end-toend workflow execution time in the presence of silent errors. As an illustration of this approach, we use a spatial outlier detection approach in staging to detect errors introduced in data transfer and storage. We also provide a CPU-GPU hybrid staging framework for error detection in order to achieve faster error identification. We have implemented our approach within the DataSpaces staging service, and evaluated it using both synthetic and real workflows on a Cray XK7 system (Titan) at different scales. We demonstrate that, in the presence of silent errors, enabling error detection on staged data alongside a checkpoint/restart scheme improves the total in-situ workflow execution time by up to $22 \%$ in comparison with using checkpoint/restart alone.
\end{abstract}

\section{KEYWORDS}

Fault Tolerances, Silent Data Corruption, Error Detection, In-situ Workflows, Data Staging

\section{ACM Reference Format:}

Shaohua Duan, Pradeep Subedi, Philip E. Davis, Manish Parashar. 2019. Addressing Data Resiliency for Staging Based Scientific Workflows. In The International Conference for High Performance Computing, Networking, Storage, and Analysis (SC '19), November 17-22, 2019, Denver, CO, USA. ACM, New York, NY, USA, 12 pages. https://doi.org/10.1145/3295500.3356158

Permission to make digital or hard copies of all or part of this work for personal or classroom use is granted without fee provided that copies are not made or distributed for profit or commercial advantage and that copies bear this notice and the full citation on the first page. Copyrights for components of this work owned by others than ACM must be honored. Abstracting with credit is permitted. To copy otherwise, or republish, to post on servers or to redistribute to lists, requires prior specific permission and/or a fee. Request permissions from permissions@acm.org.

SC '19, November 17-22, 2019, Denver, CO, USA

(C) 2019 Association for Computing Machinery.

ACM ISBN 978-1-4503-6229-0/19/11 . \$ \$15.00

https://doi.org/10.1145/3295500.3356158

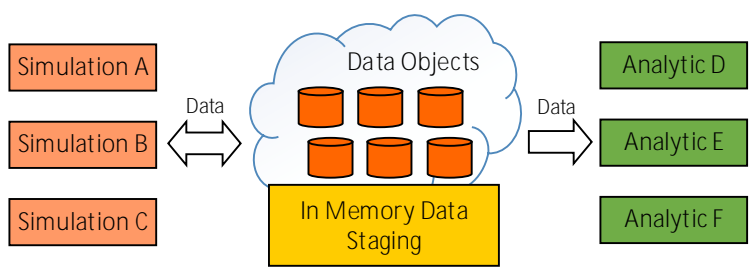

Figure 1: A typical data staging workflow.

\section{INTRODUCTION}

Scientific workflows running on emerging extreme-scale systems can provide new insights into some of the most important problems in science and society, such as those being addressed by the US Exascale Computing Program (ECP) [26]. However, extreme scales also lead to new challenges in areas such as data management, resilience, and energy efficiency. Research and innovation to address these challenges is ongoing at all levels, from hardware architectures and system software to algorithms and applications.

In-situ workflow approaches, such as those based on data staging and in-situ/in-transit data-management, have emerged as effective solutions for addressing data-related challenges at extreme scales, and are being adopted by applications across current high-end computing systems $[11,21]$. The data staging area, i.e., a set of additional compute nodes allocated by the user when launching an application workflow, leverages resources (i.e., compute and memory) on the HPC system itself to support the interaction and data couplings required by the workflows as well as to execute data-processing components of the workflow close to where the data is being produced. This reduces the amount of data that needs to be moved off the system, for example to persistent storage [4]. One example of this is the coupled multi-scale, multi-physics turbulent combustion application S3D [9]. S3D has an intricate data-processing workflow that includes multiple analyses performed at different temporal frequencies on non-overlapping subsets of data, and staging-based in-situ frameworks such as DataSpaces [13] have been used effectively to support such workflows. Figure 1 illustrates a coupled simulation workflow, wherein the primary scientific simulation is the data producer and the data consumer(s) include secondary simulations, analytics services, and/or visualization applications coupled to the producer.

Recent research has also addressed resilience at extreme scales and the expected higher rates of fail-stop failures and silent errors (also called silent data corruption) [8]. These works have explored techniques for minimizing application vulnerability to such failures. However, the impact and remediation of such failures and errors on in-situ workflows has only recently been explored. The impact of failures and errors can potentially be amplified in the 
case in-situ workflows due to the dependencies, interactions and data exchanges between the components of the workflow. While our previous work [15] has tried to minimize the impact of fail-stop failures on staging-based in-situ workflows, the impact and remediation of silent errors specifically on such in-situ workflows has not been explored to the best of our knowledge.

A silent error is an unintentional change to a bit in memory. These undetected bit flips impact the correctness and performance of applications and workflows [8]. These types of error events are already present and impactful in high-end computing. For example, silent errors have been observed to occur approximately once per day on Jaguar, located at Oak Ridge National Laboratory (ORNL), which is a 18688-node supercomputer and each node is equipped with $16 \mathrm{~GB}$ of DRAM supercomputer $[18,19]$. More recently, a detailed analysis of DRAM and SRAM faults on Hopper, a 6000-node supercomputer located at Lawrence Berkeley National Laboratory (LBNL), indicates that Hopper encounters $~ 32$ FITs (failures per billion hours of operation) per DRAM device [22]. As systems approach extreme scales, these error rates will grow to become unacceptably high, leading to an increase in silent data corruption rates. If we expect the per-component fault rates in an extreme scale system to be similar to those observed in current devices, the mean time between undetected memory errors for the system as a whole will be on the order of minutes. Hardware-level error detection approaches alone, such as error-correcting codes (ECCs), cannot cover all silent errors and additionally need to cooperate with system-level and application-level error detection approaches to achieve maximally reliable system design $[16,20]$.

Although various error-detection techniques for silent errors, such as ABFT [7] and time-series predictions [6], have been widely studied, these studies have generally been in the context of single applications rather than workflows, which are a composition of multiple interacting component applications. As extreme scale workflows are often long-running and the final result of the workflow dependends upon intermediate results, a silent error or data corruption in any component can invalidate the entire execution of the workflow, with substantial impact. As a result, it is important to detect and isolate silent errors in a component application as early as possible and to contain the propagation of these errors between components.

To that end, this paper explores the detection and remediation of silent errors for staging-based in-situ workflows. In this research, we leverage the fact that such in-situ workflows utilize data staging frameworks [13][14] to exchange data, and use idle computational resources within the data staging area to provide uniform and efficient error detection. Note that the paper does not focus on a specific error detection approach nor propose a new approach, but rather focuses on how existing approaches can be effectively used to provide data resiliency for staging-based in-situ workflows. Specifically, we perform data validation as soon as the data is written to the staging area. If an error is identified in recently written data, the faulty application is instructed to roll back to the last known correct checkpoint and re-execute. In this way, the application waiting to consume the written data will only get access to the correct data, and error propagation from producer to consumer is eliminated. We use a spatial outlier detection method for data validation as an illustrative example in this paper, and also explore how the performance impact of data verification can be reduced by leveraging GPU resources at the staging nodes to execute the error detection algorithms.

We have implemented our staging based error-detection framework for in-situ workflows within DataSpaces [13] and have deployed it on the Titan Cray XK7 production system at Oak Ridge National Laboratory (ORNL). We then have evaluated its effectiveness and performance using using both synthetic workloads and the S3D combustion workflow. These evaluations demonstrate that enabling error detection in the staging area can efficiently detect silent errors for various use cases, sustaining performance and scalability in spite of frequent silent errors.

The rest of the paper is organized as follows. In Section 2, we discuss background and motivation related to staging-base silent error detection for in-situ workflows as well as error detection approaches, and introduce our staging-based error detection framework in Section 3. In Section 4, we evaluate our approach using various synthetic and real-world scientific simulation workflows. Section 5 provides details of various related work, and the paper is concluded in Section 6.

\section{BACKGROUND AND MOTIVATION}

Each silent error is a potential threat to the data integrity of an HPC application. Silent errors may occur in the form of transient bitflips and are typically caused by electronic noise or strikes by high energy particles, such as cosmic rays or proton radiation. Although the mean time between silent errors (which we will denote as MTBE in this paper) of individual components is high, at extreme scales the aggregate MTBE of the entire system is low due to the large number of system components. Additionally, silent errors are becoming more prevalent in HPC systems as lower power chips with smaller feature sizes are being deployed. Research has shown that there exists a strong inverse correlation between the spontaneous error rate and the device sizes and operating voltages [17]. Compounding the issue, scientific workflows, due to their complexity and long execution times, tend to encounter interruptions or obtain invalid results caused by silent errors at a relatively high rate. Therefore, data resiliency is a critical concern for scientific workflows running on extreme scale systems and efficient detection of silent errors is important for guaranteeing data resilience.

To this end, we make no assumptions about the source of the errors and they can come either from the hardware (error that are not correctable by ECC) or from the software due to incorrect programming logic. This paper focuses on silent errors that corrupt one or multiple bits of a floating-point scientific dataset silently, regardless of their origin, during the execution of the workflow.

\subsection{Motivating Application Workflow}

Extreme scale in-situ scientific workflows involve interactions and data exchanges between the various components composing the workflow. For example, consider the coupled combustion simulation DNS-LES workflow. S3D [9] is a massively parallel computational fluid dynamics (CFD) solver that performs first principles based "direct numerical simulations" (DNS) of turbulent combustion. DNS is very expensive both in terms of flops and data generation, since 


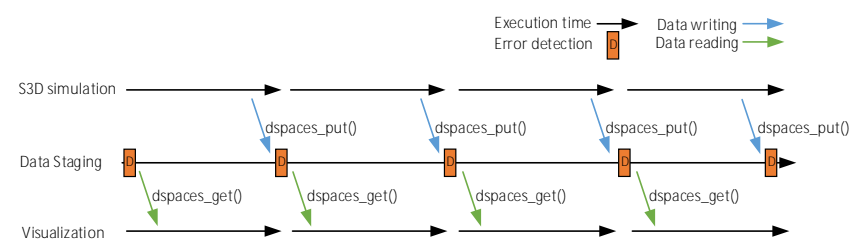

Figure 2: Coupling and data-exchange patterns for the in-situ S3D coupled simulation workflow.

it resolves the entire range of spatial and temporal scales in the continuum regime of a given problem. Large Eddy Simulation (LES) simulates a combustion environments at a lower resolution, resulting in better performance for simulating features that can tolerate this lower resolution. Many problem classes involve features that cannot be performantly and accurately simulated by DNS or LES alone, but can be effectively simulated by a coupled solution, requiring in-situ coupling of two different solvers running at different resolutions. Furthermore, knowledge discovery and visualization from a S3D simulation run can be a daunting task due to the size of the data set generated and the complexity of the temporally evolving intermittent phenomena. To understand the correlation of scalar fields such as temperature, mixing rates and species concentrations in turbulent flames, simulation-time feature extraction and visualization is necessary.

The S3D workflow consists of S3D simulation and visualization coupling application, as illustrated in Figure 2. In each coupling cycle, the workflow first executes S3D simulation for several time steps, moves the data generated to the staging area, which is the processed by the analytics/visualization applications for feature extraction.

When the workflow passes data between the S3D simulation components and the visualization components, dozens of 3D scalar and vector field components (fluid velocity, molecular species concentrations, temperature, pressure, density, etc) are transferred using staging or other mechanisms. If there are silent errors in this data, these errors will also be propagated to the coupled components of the workflow. This will result in erroneous results in the visualization and analysis products. Even worse, in the case of multiphysics coupling, even small errors can have catastrophic results, especially to the stability of non-linear PDEs. These errors have the potential to significantly alter the accuracy of the simulation, and may not be otherwise detected by the simulation without expensive analysis on the invariant terms of the simulation.

To prevent such silent errors from propagating across components, we must identify and recover from data corruption before the data transfer is completed. While error detection algorithms can be hard-coded into applications and invoked before every data exchange, this can become quite expensive. In the case of stagingbased in-situ workflows, data transfers between workflow components is done through staging, which provides an opportunity to leverage computational capabilities on the staging resources to perform error detection only on the exchanged data in an asynchronous manner. In this paper, we explore the feasibility add effectiveness of such an approach.

A further motivation for staging-based error detection is that simulation and analysis applications in workflows are commonly

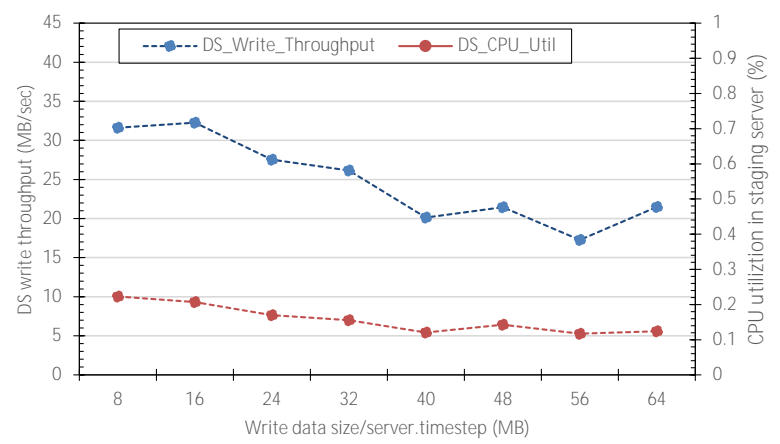

Figure 3: Cumulative data write throughput (blue dot line) and CPU Utilization = compute time in staging/write response time (red line) for different problem sizes when writing the entire data domain to data staging.

CPU-bound, so frequently performing error detection in such applications will significantly degrade the overall performance of the workflow. In contrast, we observed that staging resources tend to be relatively less heavily loaded compared to the computations nodes that run the workflow components. The indexing and datatransformation tasks associated with providing a data staging service are not generally CPU-intensive. Given this resource-usage pattern, it is reasonable to offload error detection task to data staging, which enables efficient error detection without suffering performance degradation. To demonstrate this, we performed experiments using a synthetic workflow on the Titan Cray XK7 system. The data used in this workflow is based on the S3D access patterns shown in Figure 2. 64 synthetic simulation processes wrote a set of $\mathrm{n}$-dimensional arrays to 4 staging servers with varying staged data sizes in each time step. The arrays consist of uniform double-precision floating point data.

The amount written to each staging server per time step varied between $8 M$ and $64 M$ and total data size stored in the staging area through the life of the workflow varied between $320 M$ and $2560 M$. Using this configuration, we measured the average write throughput and CPU utilization for the data staging servers under different workloads. The results are plotted in Figure 3. As the size of the workload was increased, the CPU utilization of the staging cores remained consistently low, while the write throughput has already reached the peak and decreased. In this particular test, the maximum CPU utilization was observed around $22 \%$ when the write size was $8 M$ for each staging server per time step. This experiment illustrates that the compute resource in the staging area is not fully utilized and the staging performance is constrained by other resources limitation such as network congestion. Therefore, we have an opportunity to leverage compute resource within stagingarea to perform error detection without degrading the read and write throughput of the staging service.

\subsection{Silent Error Detection Techniques}

Theoretically, any error detection approach at the software level is fit for a staging-based error detection framework. In this section, we will briefly talk about integrating three widely-used error detection approaches in data staging and figure out generality, accuracy and performance of each approach. The accuracy of error detection is 
quantified by using two measures: recall and precision, which are defined as:

$$
\begin{gathered}
\text { recall }=\frac{\text { TruePositives }}{\text { TruePositives }+ \text { FalseNegatives }} \\
\text { precision }=\frac{\text { TruePositives }}{\text { TruePositives }+ \text { FalsePositives }}
\end{gathered}
$$

2.2.1 Process Replication. Process replication [18] has been widely used for tolerating both silent errors and fail-stop failures. It creates replica tasks, such as MPI process, for each primary task and compares the computation results between replicated processes to detect corrupted data. This approach is very general, has high recall and precision. Unfortunately, process replication may not always be feasible because computing redundant tasks in the staging area will impose large overheads and significantly degrade throughput of the data request, since the compute resources in data staging are much smaller than that in application components.

2.2.2 Algorithm-Based Fault Tolerance. The general idea of AlgorithmBased Fault Tolerance (ABFT)[7] is to introduce information redundancy in the data, and maintain this redundancy during computation. Linear algebra operations over matrices are well-suited to apply such a scheme: the matrix (original data of the user) can be extended by a number of columns, in which checksums over the rows are stored for future data verification. It imposes a low overhead on the computation and guarantees good recall in general. The disadvantage of integrating ABFT in data staging is that this technique has only been implemented for a limited set of linear algebra application, and so is only available to a small subset of the vast spectrum of scientific applications.

2.2.3 Outlier Based Error Detection. Most of the datasets produced by HPC scientific applications have expected informational characteristics that reflect the properties of the underlining physical phenomena that the applications seek to model [20]. Silent data corruption via bit flips alters the value of the data causing it to deviate from these standard characteristics. Therefore, corrupted data can be detected as an outlier based on its deviation from the expected range of normal values. There are two classes of outlier detection: time-series outlier detection and spatial outlier detection. Time-series outlier detection [6] is illustrated in Figure 4(a). HPC scientific application often iteratively operate upon data, changing their values over time. At each iterative time-step, a time-series outlier detection approach can be used to dynamically predict the possible range for data values at the next time-step, and a data value can be considered as an outlier if it falls outside this range.

Spatial outlier detection [3] is illustrated in Figure 4(b). A spatial outlier is a spatially-referenced object whose non-spatial attribute

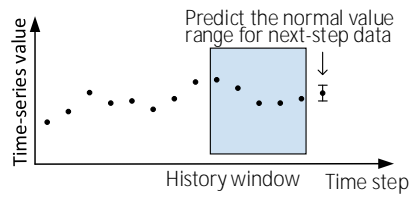

(a) Time-series outlier detection

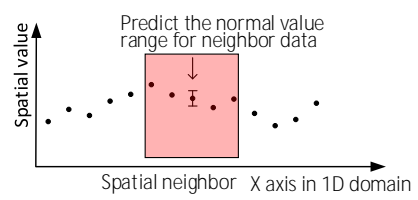

(b) Spatial outlier detection
Figure 4: Examples of time series and spatial outlier detection techniques for a 1D data domain. values are significantly different from those of other spatially referenced objects in its spatial neighborhood. A spatial neighborhood may be defined based on spatial attributes, e.g., location, using spatial relationships such as distance or adjacency. Non-spatial attributes between spatially referenced objects can be compared within that neighborhood, considering those values whose nonspatial attributes deviate significantly to be spatial outliers. Although not perfectly accurate, outlier detection techniques can still detect a substantial fraction of silent errors, and more importantly these methods incur low overhead. These properties make them attractive candidates for staging-based error detection.

Although outlier-based error detection approaches are good candidates for SDC-detection, it should be noted that the choice of the most appropriate error-detection approach depends on the data characteristics of the workflow; our work aims to provide a pluggable approach for enabling error-detection in the staging area and to highlight its benefits, and can use the chosen most appropriate error-detection approach for a specific workflow.

\section{ERROR DETECTION IN STAGING}

In this section, we first model our staging-based error detection framework. We then analyze it by simulating scientific workflow events and present factors that influence the rate of error detection in staging. Finally, we propose optimizations for implementing error detection approaches in the data-staging area and leveraging GPU resource (if available) to ameliorate the impact of performing error detection on common $\mathrm{I} / \mathrm{O}$ operations.

\subsection{Modeling Error Detection in Staging}

Notations: Let $C, T_{c}$, and $T_{s}$ denote the cost of checkpointing, the optimal time cycle for checkpointing, and the time cycle of error detection in staging, respectively. Let $R_{s}$ represent the recall of error detection in staging, which is the proportion of detected errors over all errors that have occurred in the course of execution. If $D$ is the cost of error detection in a given checkpoint component and $D_{s}$ is the cost of error detection in staging, we assume that $D_{s}<<D$, since the amount of data exchanged via staging area is much smaller than checkpoint data size. $W_{\text {base }}$ denotes the base execution time of a workflow without any resilience technique. The notations that are used in the model are listed in Table 1.

\begin{tabular}{|c|l|}
\hline Symbols & Explanation \\
\hline$C$ & Cost of checkpointing \\
\hline$T_{c}$ & Optimum time cycle for checkpoint \\
\hline$D$ & Error detection cost in the checkpointing component \\
\hline$T_{s}$ & Time cycle for error detection in data staging \\
\hline$D_{s}$ & Error detection cost in data staging \\
\hline$T_{r}$ & $\begin{array}{l}\text { Time interval from last checkpoint to the error detection in } \\
\text { data staging }\end{array}$ \\
\hline$R_{s}$ & recall of error detection in data staging \\
\hline$P_{s}$ & precision of error detection in data staging \\
\hline$P_{f}$ & Probability of silent errors during execution time \\
\hline$T_{w}$ & Time cycle for workflows pattern \\
\hline$W_{\text {base }}$ & $\begin{array}{l}\text { Workflow execution time without resilience techniques and } \\
\text { failures }\end{array}$ \\
\hline
\end{tabular}

Table 1: Symbols summary 


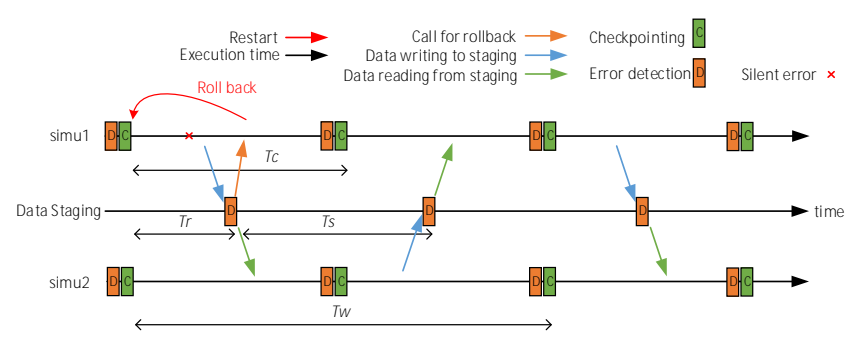

Figure 5: An illustration of a typical workflow with two coupled simulations simu1, simu2. Simulations alternate in exchanging data via data staging. For fault tolerance, each simulation performs checkpointing and silent error detection tasks based on their optimal checkpoint time cycles.

Fault tolerance model: Scientific workflows typically employ a checkpoint/restart mechanism to periodically perform checkpointing tasks to enable rollback to the previous state in case of a fail-stop failure. To deal with both fail-stop failures and silent errors, checkpointing tasks are coupled with error detection (or verification) mechanisms. To guarantee that checkpoint data is error-free, error detection is performed just before checkpointing tasks [5]. If the verification fails, it can be assumed that a silent error has occurred and the application must rollback to the previous checkpoint for error-free data.

Figure 5 shows a typical workflow with a checkpointing scheme combined with error detection. In each checkpoint cycle, coupled simulations simu1, simu 2 can access staging servers and fetch or write data from/to the servers. Since data is stored in the staging area, we can improve the fault tolerance scheme by leveraging idle compute resource in the staging area to perform data verification. In this way, during each checkpoint cycle, there will be multiple error detections in the staging for early detection of silent errors and to save re-execution time. For the checkpointing scheme, a workflow makes a global checkpoint and performs error detection based on its optimal checkpoint time cycle for fail-stop failures. In the following analysis, we compare the approach where error detection occurs only at checkpoint-time with the approach that performs error detection in both staging and at the checkpoint. The goal of performing error detection in staging is to minimize the expected execution time of the workflow, $E(W)$.

3.1.1 Error detection in checkpoint component. Suppose that the probability of a silent error happening in the workflows is $P_{f}$. Let $T_{D C}$ be the maximum cost for error detection and checkpoint of simu1, simu2: $\operatorname{Max}\left(D_{1}+C_{1}, D_{2}+C_{2}\right)$. Let $T_{c C}$ denote the maximum time chunk for two consecutive checkpoints: $\operatorname{Max}\left(T_{c 1}-C_{1}, T_{c 2}-C_{2}\right)$. Also, error detection in the checkpoint component can always fully verify data. Then, the expected execution time of the workflow $\left(E\left(W_{c}\right)\right)$ can be computed as:

$$
E\left(W_{c}\right)=\frac{T_{w}}{T_{c}}\left(T_{D C}+P_{f} \times T_{c C}\right)+W_{\text {base }}
$$

3.1.2 Error detection in checkpoint and staging. We now add an extra error detection in the staging area. Firstly, let us suppose that any silent error happening during $T_{s}$ can be detected. That means recall and precision for error detection in staging are $R_{s}=$ $100 \%, P_{s}=100 \%$. In this scenario, the workflow executes for an extra $D_{s}$ time until error detection in staging completes and sends an error notification back. Then, the workflow needs to rollback to the previous error-free checkpoint and discard $T_{r}+D_{s}$ time worth of work. Therefore, the expected execution time of the workflow $\left(E\left(W_{s}\right)\right)$ can be computed as:

$$
E\left(W_{s}\right)=\frac{T_{w}}{T_{c}}\left(T_{D C}+P_{f}\left(T_{r}+D_{s}\right)\right)+W_{\text {base }}
$$

Then, we can make our model more realistic by only assuming that the precision of error detection in staging $P_{s}=100$. In this case, the staging can detect $R_{S}$ percent of silent errors and the remaining $1-R_{s}$ percentage will be detected by the checkpoint component. Thus, the expected execution time of the workflow $\left(E\left(W_{s}\right)\right)$ can be updated to:

$$
\frac{T_{w}}{T_{c}}\left(T_{D C}+P_{f}\left(\left(1-R_{s}\right) T_{c C}+R_{s}\left(T_{r}+D_{s}\right)\right)\right)+W_{\text {base }}
$$

When the recall rate is $R_{s}$ and the precision is $P_{s}$ for the error detection in staging, the staging will generate $1-P_{s}$ percentage needless rollback operations due to the introduction of false positives in detection. The penalty for false positives is $T_{p}=\left(1-P_{s}\right)\left(T_{r}+D_{s}\right)$. Thus, the expected execution time of the workflow $\left(E\left(W_{s}\right)\right)$ can be updated to:

$$
\frac{T_{w}}{T_{c}}\left(T_{D C}+P_{f}\left(\left(1-R_{s}\right) T_{c C}+R_{s}\left(T_{r}+D_{s}\right)+T_{p}\right)\right)+W_{\text {base }}
$$

From the above equations, it can be expected that adding error detection in the staging area can reduce the total workflow execution time in the presence of errors, which can be computed as:

$$
E\left(W_{c}\right)-E\left(W_{s}\right)=\frac{T_{w}}{T_{c}} P_{f}\left(R_{s}\left(T_{c C}-T_{r}-D_{s}\right)-T_{p}\right)
$$

\subsection{Simulation and Analysis}

In order to determine the expected advantage of this approach in practice, we simulate the execution of a scientific workflow using the parameters (i.e., $M T B F, C$, and $T_{c}$ ) suggested in [5], which are also listed in Table 2. For MTBF and MTBE, we calculate the $M T B F$ and $M T B E$ of one computing node, which is 4.3 years for fail-stop failures and 7.9 years for silent errors. The overall $M T B F$ and $M T B E$ are obtained by dividing the per-node $M T B F$ and $M T B E$ by the total number of nodes running the workflow. For example, when 65536 nodes are used in extreme scale systems, the overall $M T B F$ and $M T B E$ are $2064 s$ for fail-stop failures and $3784 s$ for silent errors. The silent errors are generated by following an exponential distribution of parameter $\lambda=\frac{1}{M T B E}$. The disk checkpoint $\operatorname{cost} C$ is close to $300 s$, which is typical of many state-of-the-art platforms, such as Hera [5]. Under the assumed MTBF for fail-stop failures and the checkpoint $\operatorname{cost} C$, we get the optimum checkpoint cycle of $1491 s$ through Daly's formula [25]. Furthermore, we assume that there is no relation between $M T B F$ and $M T B E$, and $M T B F$ will be kept constant in the simulation test cases. For error detection, we assume that the error detection in a checkpoint has a cost of $100 \mathrm{~s}$ with the recall and precision of $100 \%$ and that error detection in staging has a cost of $4 s$ with a recall $70 \%$ due to error detection being performed on a subset of the global data domain. We assume that the computation and communication cost of the workflow is 81920s (22.6hr), which does not include checkpointing and error detection tasks. 


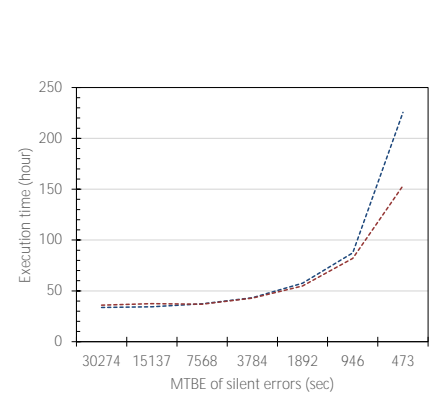

(a) Simulation case 1

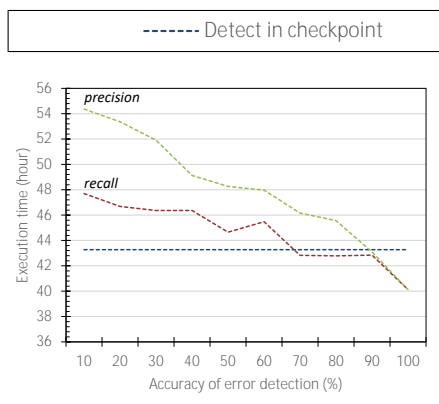

(b) Simulation case 2

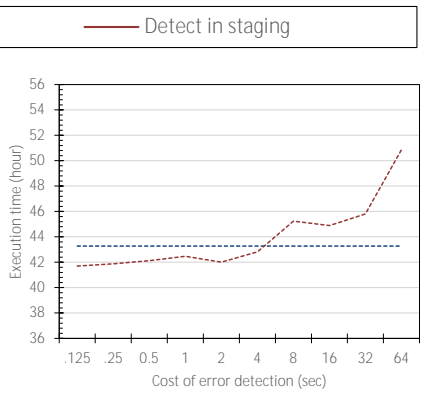

(c) Simulation case 3

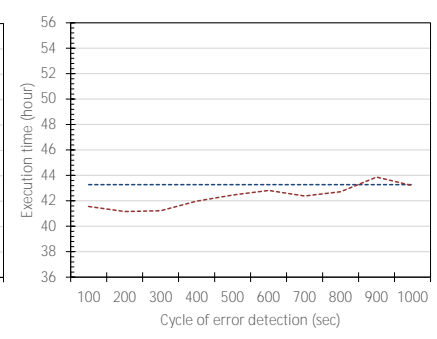

(d) Simulation case 4

Figure 6: The simulated total work flow execution time comparison between error detection in checkpoint component (blue dot line) and error detection in both checkpoint and staging (solid line).

\begin{tabular}{|c|c|c|c|c|}
\hline No. of case & Case 1 & Case 2 & Case 3 & Case 4 \\
\hline$M T B E$ & $/$ & $3784 s$ & $3784 s$ & $3784 s$ \\
\hline$C$ & $300 s$ & $300 s$ & $300 s$ & $300 s$ \\
\hline$D$ & $100 s$ & $100 s$ & $100 s$ & $100 s$ \\
\hline$T_{c}$ & $1491 s$ & $1491 s$ & $1491 s$ & $1491 s$ \\
\hline$P_{s}$ & $100 \%$ & $/$ & $100 \%$ & $100 \%$ \\
\hline$R_{s}$ & $70 \%$ & $/$ & $70 \%$ & $70 \%$ \\
\hline$D_{s}$ & $4 s$ & $4 s$ & $/$ & $4 s$ \\
\hline$T_{s}$ & $600 s$ & $600 s$ & $600 s$ & $/$ \\
\hline$T_{w}$ & $81920 s$ & $81920 s$ & $81920 s$ & $81920 s$ \\
\hline
\end{tabular}

Table 2: The parameter configuration for workflow time sequence emulation.

Table 2 describes the inputs of the emulation program. The emulation results are presented in Figure 6, followed by a detailed discussion and analysis of each. The reported results are the average of 100 experimental runs.

In simulation case 1, where we vary the workflow's $M T B E$, our approach has better performance than performing error detection only in checkpoint as long as MTBE is under 3784s. We performed analysis for different values of recall under precision $=100 \%$ and different values of precision under recall $=100 \%$ in simulation case 2. It can be seen that when recall is greater than $70 \%$ or precision is greater than $89 \%$, error detection should be performed in the staging area. For the cost of error detection when it is less that $4 \mathrm{~s}$, error detection in staging outperforms the detection in checkpointing, which is illustrated in simulation case 3 . To analyze the impact of $T_{s}$, we also varied $T_{s}$ in simulation case 4 and observed that when $T_{s}<800 s$, our approach is beneficial and it maintains similar performance for higher values.

Based on Equation 3 and the analysis above, we can deduce that the advantage of enabling error detection in the staging depends on the following factors: ( $i$ ) The frequency of silent errors MTBE (higher will see more advantage from the in-staging approach); (ii) The recall and precision of error detection in staging $R_{s}, P_{s}$ (higher is better); (iii) The cost of error detection in staging $D_{s}$ (lower is better); (iv) The frequency of staging operation $T_{s}$ (performance will improve with less frequent staging operations).

In addition, unlike recall, lower precision can significantly deteriorate the performance of error detection in staging. Part of the reason for this is that lower precision indicates high false positive, which can unnecessarily rollback the workflow frequently. In contrast, lower recall corresponds to high false negative, which means that CPU cycles are wasted performing error detection that is unable to detect silent errors. Generally, the cost of error detection in staging is much less than the cost of re-executing the workflow.

\subsection{Implementing Error Detection in Staging}

While the integration of error detection within a data staging framework seems straight-forward, naïve application of error detection technique in the data staging can result in significant performance degradation and low error detection accuracy. One of the challenges for error detection in staging is that the data staging framework stores data temporarily during data exchanges, so an entire spatial dataset is not always available. Another challenge for error detection in staging is that data staging has relatively limited compute resource, so an error detection approach having a long computation time could significantly degrade the performance of staging servers. Thus, an ideal error detection approach should maintain high accuracy, especially high precision, in error detection. Also, the approach should have light overhead and minimal impact on common data staging operations, such as data put() and get().

In the following section, we introduce two optimization techniques which can achieve the twin goals of lightweight execution and high accuracy. We use Spatial Local Outlier Measure (SLOM) [24] as an example to illustrate how to integrate an error detection approach in the data staging efficiently. SLOM is a type of spatial outlier detection approach that can capture both spatial auto-correlation (non-independence) and spatial heteroscedasticity (non-constant variance), which are common features of scientific spatial datasets. In SLOM, the effects of spatial auto-correlation are factored out by a measure $d(o)$. The variance of a neighborhood is captured by $\beta(o)$, which quantifies the oscillation and instability of an area around $o$.

$$
\operatorname{SLOM}(o)=d(o) * \beta(o)
$$

3.3.1 CPU-GPU Hybrid Staging. In an extreme-scale workflow, staging servers need to process thousands of requests. Thus, any delay caused by error-detection will interfere with servicing other requests, amplifying the impact, and potentially causing a significant delay across the whole workflow. To minimize this impact, we propose offloading workload to GPUs, when available. GPUs are now widely used as general purpose devices in HPCs. They enable significant speed-ups in performance for scientific computation and analysis tasks. Since silent error detection approaches are computation intensive tasks, we can leverage these GPUs to achieve 
significant acceleration of error detection. Specifically, CPU cores in the dedicated staging node can be used to serve communication and regular staging operations, such as indexing and data storage, while the data from local CPU memory is offloaded to GPUs and GPU kernel performs the spatial outlier detection.

There are two main challenges in processing large amounts of data on the local GPU device: relatively limited bandwidth and small memory size. We address these issues as follows. The data staging process first partitions the larger geometric dataset into several smaller tiles, such that each tile fits into the GPU global memory. Next, the tiles are stored in a queue, which is located in CPU memory and is ready to be offloaded to GPU global memory. While the GPU threads process the tile, the next tile in the queue is prefetched into GPU global memory to hide the data transfer latency. This CPU-GPU hybrid staging approach can also be leveraged by CPU-only application workflows, such as the S3D simulation workflow. The pseudo code of spatial outlier error detection in CPU-GPU hybrid staging is provided in Algorithm 1. This can be easily extended to other error detection methods.

3.3.2 Tuning precision and recall in Staging. Although recall and precision both play a very important role for error detection in staging, from section 3.2, we can deduce that compared with recall, a lower precision can significantly deteriorate the performance of error detection in staging. In addition, many outlier-based error detection approaches output a score quantifying the level of 'outlierness' of each data point, but this value does not provide a concise summary of the small number of data points that should be considered as silent errors. Specifically, in SLOM simply identifying the data point with the maximum SLOM value as a silent error will virtually always produce a false positive.

In the following, we introduce dataset/feedback training which can tune precision and recall for outlier based error detection approaches so as to minimize false positives with acceptable false negatives. During the dataset training, we inserted synthetic bit flip errors into the staged dataset and monitored the minimum value that can just cover all synthetic errors, then we mark it as

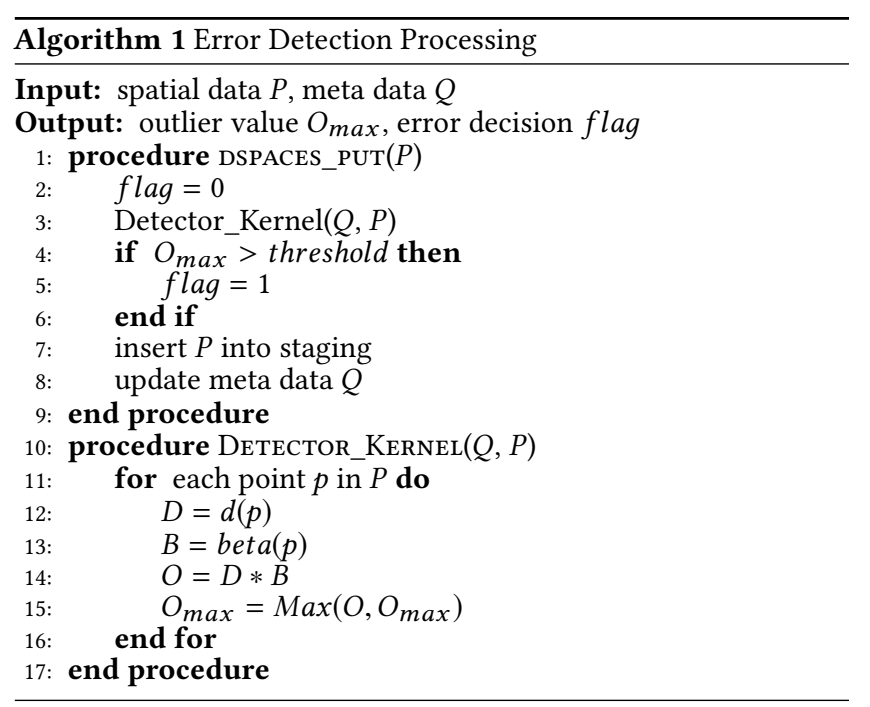

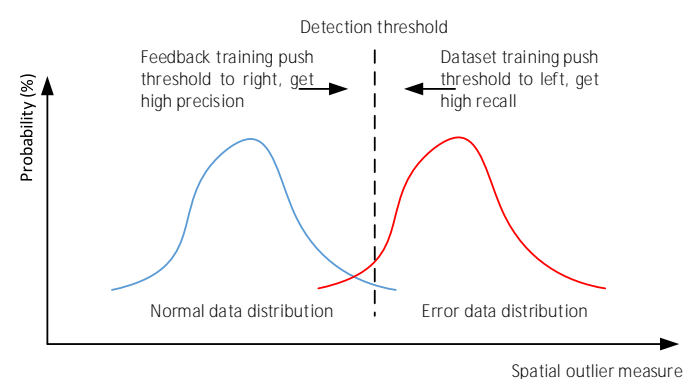

Figure 7: An illustration of the behavior of and relation between dataset training and feedback training.

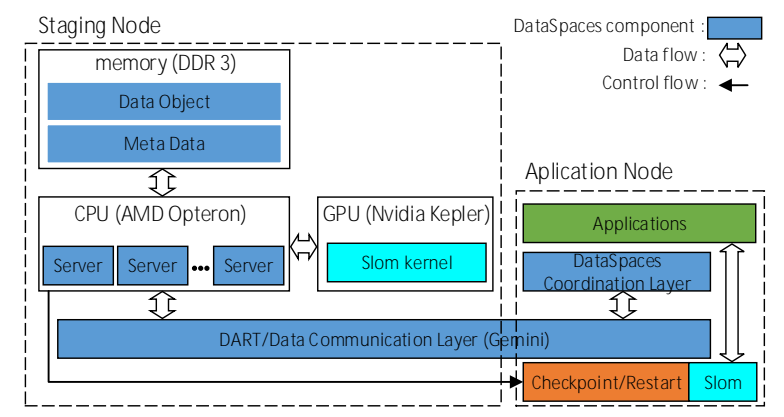

Figure 8: Implementation of error detection in CPU-GPU hybrid staging

the error detection threshold. For simplification, in our implementation the synthetic silent errors are uniformly distributed along the dataset and bit position. The threshold may be different for different simulation time steps, datasets, and staging servers.

During the feedback training, at the initialization of runtime, staging servers systematically calculate a SLOM value for each data point and keep track of the maximum observed SLOM value, as error detection threshold threshold. The staging servers then start to perform error detection for the available dataset. When the staging server finds one silent error candidate and the workflow is re-executed from the last checkpoint, it goes into feedback training mode. The staging server compares the new SLOM value with the previous one which was detected as an error. If the new SLOM value in that area is under the threshold, that means the staging server accurately identified the silent error. Otherwise, the previously detected silent error is a false positive and threshold is raised. Figure 7 illustrates that feedback training tunes the detection threshold towards the error data distribution and achieves higher precision of error detection. Similarly, dataset training pushes the detection threshold toward a normal data distribution and achieves higher recall.

\section{EXPERIMENTAL EVALUATION}

In this section, we evaluate staging based error detection framework, implemented as an extension of DataSpaces [13], an open-source data staging framework, and perform evaluations using both real and synthetic applications in the presence of silent errors.

In Section 3, we described our model for error detection in staging. In this section, we provide a specific implementation for the model on Titan HPC system. Figure 8 shows the overall system 


\begin{tabular}{|l|l|}
\hline Total number of cores & $(16$ to 128$)+16+16=48$ to 160 \\
\hline No. of writer cores & $2 \times 4 \times(2$ to 8$) \times 4 \times 4=16$ to 128 \\
\hline No. of staging cores/nodes & $16 / 1$ \\
\hline No. of reader cores & 16 \\
\hline Volume size & $64 \times 128 \times 64$ to $256 \times 128 \times 128$ \\
\hline In-staging data size (20ts) & 80 to $640 \mathrm{MB}$ \\
\hline Workflow pattern & Write immediately followed by Read \\
\hline Cycle for error detection & 1 time step \\
\hline
\end{tabular}

Table 3: Experimental setup for performance tests.

configuration for error detection in the workflow. In the Titan Cray XK7 system, each compute node contains a 16-core 2.2GHz AMD processor with 32 GB of DDR RAM, a NVIDIA Kepler accelerator (GPU) with 6 GB of GDDR RAM and a Cray Gemini high-speed interconnect. Although subsequent experiments are only run on Titan, recently deployed machines such as Summit (a 4,608-node supercomputer at Oak Ridge National Laboratory (ORNL)) also have GPUs attached to each nodes and our approach can benefit from these GPUs. Please note that our implementation of SLOM using CPU-GPU is just an example of an error detection method. The goal of the paper is to leverage idle staging resources to perform error detection using the error detection method of choice for staging based in-situ scientific workflows. The system architecture permits a key component: dedicated staging nodes, distinct from the application nodes. Simulation and analysis applications' processes run on application nodes. Before an application process checkpoints its entire data into the parallel file system, the process also performs an error detection locally to ensure data correctness. The DataSpaces coordination layer in application nodes sends dataset to staging servers through the DART layer (DataSpaces' data communication layer). After a staging server stores the data in local memory, it performs error detection in the staging nodes. For CPU-GPU hybrid staging, it will offload error detection into local GPU if the GPU compute resource is available for the dedicated staging node. When a silent error is detected, the staging server sends error message to application's checkpoint/restart components and triggers a process restart. In order to do that, we update the DataSpaces interface function $d s \_$put() so that it can return the detection result back to applications and indicate if there is a silent error in the dataset.

We performed experiments that fall into four broad categories: performance experiments, synthetic test cases, and real-world large scale experiments. All staging servers run on dedicated compute nodes with GPU accelerators.

\subsection{Performance Experiments}

In this subsection, we evaluate whether applying idle resources in data staging to perform silent error detection has any adverse affect upon the applications connected to the staging servers. In order to quantify the interference (if any) caused by the proposed technique, we measure the write performance of the staging servers with error detection enabled. We also record write-response time without error detection in staging as a baseline. The setup of these experiments is described in Table 3.

Figure 9 shows the cumulative write-response time for staging severs with error detection. We can see that error detection involve much lower performance penalties even at large dataset in data staging. For example, for CPU-based error detection in staging,

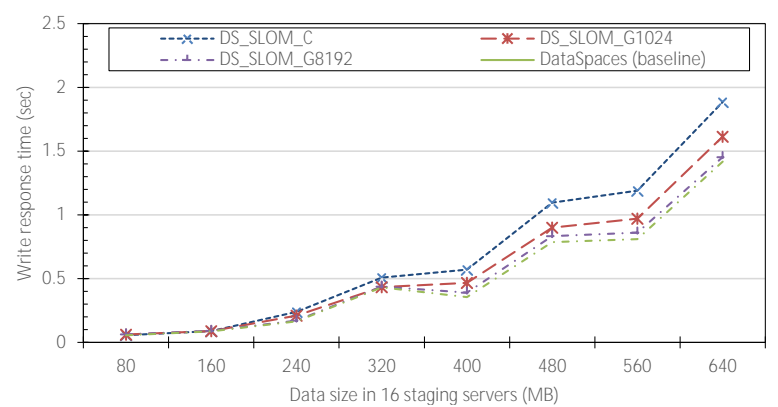

Figure 9: Comparison of the data write-response time for data staging with error detection on Titan. DataSpaces: Data staging write response time without error detection (baseline); DS_SLOM_C: Data staging write response time with error detection in CPU staging; DS_SLOM_G1024/8192: Data staging write response time with error detection in CPU-GPU hybrid staging under 1024/8192 GPU threads.

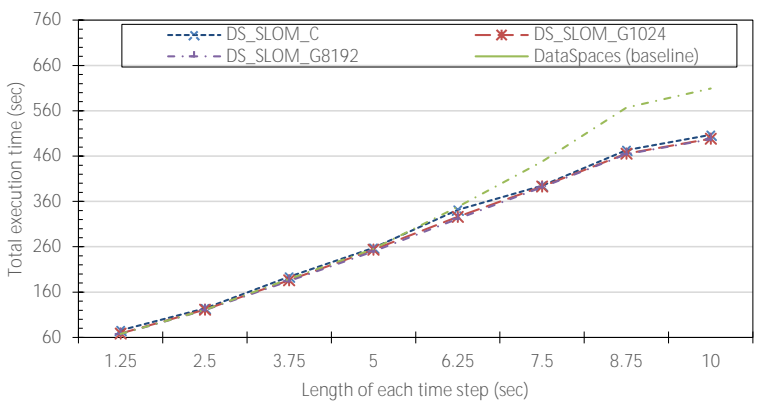

Figure 10: Comparison of the total execution time of the workflow under different error detection frequencies or length of each time step in data staging. Error detection is performed in each time step.

the write response time overhead was no worse than $24.11 \%$ as compared to the same application without error detection. When we perform error detection in CPU-GPU hybrid staging, we see much lower overhead than the CPU-alone case. The write-response time increases by only $8.41 \%$ for the 1024 GPU thread case and $2.43 \%$ in the worst case for the 8192 GPU thread case as compared to the case of no error detection. Our approach shows good overall scalability and has minimal overhead as compared to the baseline.

We also explore the overhead of error detection in staging over different workflow configurations. We plot the total execution time for a workflow with different error detection cycle in Figure 10. In this experiment, we perform error detection in staging in each time step and keep $M T B E=5$ minutes and checkpoint cycle $T_{c}=2.5$ minutes constant. We then vary the length of the time steps from 10 seconds down to 1.25 seconds. Figure 10 shows that, for larger than 5 second time steps, the benefit for error detection in staging becomes significant, with a decrease of up to $22.5 \%$ in the total execution time as compared to error detection in only the local component checkpoint. For the frequent error detection or smaller length of each time step(between 1.25 and 5 second), error detection in staging does not provide much benefit. At the extreme, for the 1.25 second time step case error detection can trigger an overhead of $11.7 \%$. 


\subsection{Synthetic Test Cases}

To better understand the performance of error detection in staging and its effectiveness, we also design synthetic cases and evaluate the total execution time of the workflow. Our synthetic experiments were performed on Titan. In these experiments, both synthetic simulation and analysis applications have their own local error detection and checkpoint component. For simplification we assume that the local error detection in checkpoint component has $100 \%$ recall and precision. We checkpoint every 10 iterations and before checkpointing dataset into the parallel file system, error detection is performed, guaranteeing that the checkpoint is error-free. Besides detecting silent errors in the local checkpoint component, the error detection is also performed through staging servers after the data is staged. We also assume that precision of error detection in data staging is $100 \%$, and recall is linearly related with the proportion of staged data. Once a silent error is detected, the workflow is rolled back to the last checkpoint and re-executed. We ran the synthetic workflow under varying proportions of staged data to total data and different frequency of error detection in staging. Then, we measure the total execution time of the workflow. In these experiments, two significant soft errors were randomly introduced into the dataset within the 40 time steps, which corresponds to $M T B E=5 \mathrm{~min}$. We re-run our experiments for 50 times and report the total execution time as an average of these runs. The set-up of these experiments is described in Table 4. The experimental results are presented in Figure 11 followed by a detailed discussion and analysis of each.

1) Case 1 - Write the entire data domain in each time step under different MTBE: Similar to simulation case 1 in Section 3.2, we vary $M T B E$ (occurrence of silent errors) between 5, 7, 10, and 20 minutes which corresponds to $100,75,50$, and 25 silent errors respectively being introduced during a total of 50 runs. Since data staging can detect silent errors and roll back workflow early in each time, the method of performing error detection in staging can get a relatively better total execution time than the error detection in local checkpoint component under the high frequent $M T B E$. This improves the total execution time of the workflow by $18.1 \%$ for $M T B E=5 \mathrm{~min}, 14.3 \%$ for $M T B E=7 \mathrm{~min}, 11 \%$ for $M T B E=10 \mathrm{~min}$ and $10.1 \%$ for $M T B E=20 \mathrm{~min}$ respectively, as compared to error detection in local checkpoint component only under correspond $M T B E$ value.

2) Case 2 - Write a subset of the data domain and perform error detection in each time step: This case is effectively a combination of simulation case 2 and 3 in Section 3.2. In this case and other subsequent cases, we inject a total of 100 errors during 50 runs of the experiment. Since different percentage of whole data-set is exchanged via staging framework, there is variance in the recall and overhead of error detection in data staging, which is based on proportion of subdomain size to the entire data domain. Thus, error detection in staging has an increase in detected errors when the amount of data in staging area increases. Our approach reduced the total execution by around $7.6 \%$ for $40 \%, 11.3 \%$ for $60 \%$ and $14.2 \%$ for $80 \%$ subset data, as compared to the error detection in local checkpoint component only.

3) Case 3 - Write the entire data domain in multiple time steps and perform error detection in each time step: This case differs from the previous case, where a certain percentage of entire data domain घxecute $n$ Checkpoint $n$ Restart $n$ Detect $n$ Re-execute

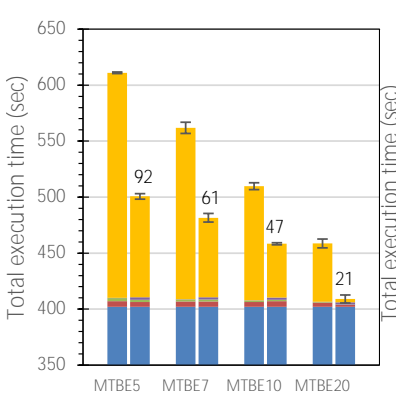

(a) Case 1

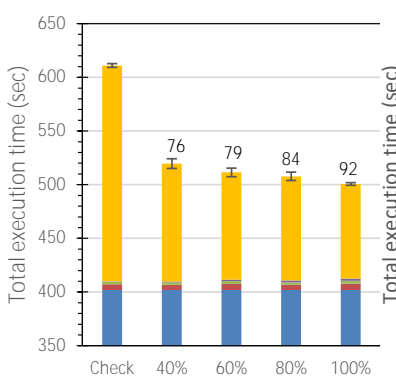

(c) Case 3

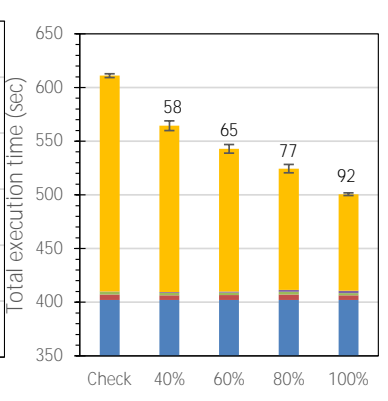

(b) Case 2
Figure 11: Breakdown of the total execution time (in seconds) for the workflows with checkpoint restart and error detection under 2 silent errors. Execute: Time spent in workflow execution; Checkpoint: Time spent to perform checkpointing; Restart: Time spent to restart workflow; Detect: Time spent performing error detection in staging; Re-execute: Time spent for re-executing the workflow from last checkpoint (correct error). The leftmost bar represents error detection in local checkpoint component only. Other bars represent error detection in both staging and local checkpoint components. Numbers on top of the bars indicate the total number of errors that was corrected by the staging component during 50 experiment runs.

was written in a time step. In this case, although a subset in written in each step, data staging can stages entire data domain in between 2 to 3 time steps. This enables staging framework to have high cumulative recall. Therefore, error detection in staging reduces the total execution time (around $14.9 \%$ for $40 \%, 16.3 \%$ for $60 \%$ and $16.9 \%$ for $80 \%$ subset data) as compared to error detection in local checkpoint component only.

4) Case 4 - Write the entire data domain and perform error detection with different time step cycle: Like simulation case 4 in Section 3.2, the entire data domain is written into the data staging periodically for every 1, 2, 4, 8 time steps separately. For error detection in staging, as the entire data is staged, a high recall of error detection is achieved. We can thus detect silent errors in the staging area and roll back the workflow immediately. Therefore, error detection in staging achieves a decrease of $2.2 \%$ for $8 t s, 8.4 \%$ for $4 t s, 13.8 \%$ for $2 t s$ and $18.1 \%$ for $1 t s$ in the total execution time relative to error detection in the local checkpoint component only. 


\begin{tabular}{|l|l|}
\hline Total number of cores & $96+16+16=128$ \\
\hline No. of writer cores & $6 \times 4 \times 4=96$ \\
\hline No. of staging cores/nodes & $16 / 1$ \\
\hline No. of reader cores & 16 \\
\hline Volume size & $192 \times 128 \times 128$ \\
\hline In-staging data size $(40$ ts) & $960 M B$ \\
\hline Workflow pattern & Write immediately followed by read \\
\hline Local checkpoint, detection cycle & $10 t s$ \\
\hline
\end{tabular}

Table 4: Experimental setup for synthetic test cases.

\begin{tabular}{|l|c|c|c|}
\hline No. of cores & 4416 & 8832 & 17664 \\
\hline No. of simulation cores & 4096 & 8192 & 16384 \\
\hline No. of staging cores/nodes & $256 / 16$ & $512 / 32$ & $1024 / 64$ \\
\hline No. of analysis cores & 64 & 128 & 256 \\
\hline Volume size & $256 \times 256 \times 256$ & $512 \times 256 \times 256$ & $512 \times 512 \times 256$ \\
\hline Data size (GB) & 110 & 220 & 440 \\
\hline Checkpoint cycle & $10 t s$ & $10 t s$ & $10 t s$ \\
\hline Detection cycle of staging & $1 t s$ & $1 t s$ & $1 t s$ \\
\hline MTBE of silent error & $300 \mathrm{sec}$ & $300 \mathrm{sec}$ & $300 \mathrm{sec}$ \\
\hline
\end{tabular}

Table 5: Configuration of core-allocations, data sizes, and data resilience for the three test scenarios on 4416,8832 and 17664 cores.

\subsection{Large Scale S3D Experiment}

We perform large scale tests of our method using the combustion DNS-LES simulation/analysis from the S3D combustion and analysis workflow [9] on Titan and compare it with error detection-only in the local checkpoint component. We also integrate error detection algorithm within S3D before checkpointing. Our staging-based error detection framework is tested using three different core counts (4416, 8832 and 17664) and corresponding grid domain sizes so that each core is assigned a spatial sub-domain of size $16 \times 16 \times 16$. In each time steps, $60 \%, 80 \%, 100 \%$ percentage of entire data passes through data staging separately. The total execution time of the workflow over 40 time steps is measured. Other core configurations, and data resilience setup are detailed in Table 5.

Figure 12 and Figure 13 illustrate the experimental results for the total execution time of S3D coupled simulation and analysis application workflow for various scale in the presence of silent errors. We also measure the precision and recall of error detection in staging. For all tests, the precision always equals $100 \%$, and the value of recall is shown on top of the bars in Figure 12 and Figure 13. For all error detection approaches, the workflow with error detection in CPU-GPU hybrid staging always shows lower execution time than the corresponding workflow running with CPU-based staging alone. Under error detection in CPU-only staging techniques with the presence of silent errors, error detection in staging reduces the total workflow execution time by up to $14.4 \%, 16.5 \%$, and $18.9 \%$ as compared to error detection in only the local checkpoint component on 4416,8832 , and 17664 cores, respectively.

The same trend can also be seen in the CPU-GPU hybrid staging. The error detection in staging improves the total execution time by up to $14.1 \%, 22.6 \%$ and $15.4 \%$ as compared to a workflow with error detection in only the local checkpoint component. In addition, when error detection is performed in staging, it was observed that the greater the fraction of the dataset being put into staging and verified, the lower the total execution time in the presence of errors. These results demonstrate that error detection in staging achieves

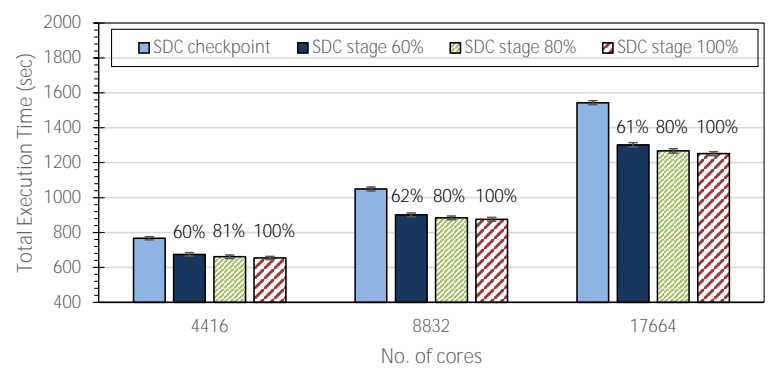

Figure 12: Comparison of the total execution time (in seconds) for the S3D simulation and coupled analysis workflow with error detection in CPU staging. Numbers on top of the bars indicate the recall of error detection in staging.

good overall scalability with small overhead for different processor counts and data sizes on extreme scale HPC systems.

Another concern for error detection in data staging is about the extra energy consumption involved in utilizing a data staging area. For this paper, we did not explore energy optimization approaches for error detection in data staging, nor conduct experiments to evaluate energy consumption. Although evaluations of energy efficiency are beyond the scope of the paper, we can make some general statements about the energy consumption of our approach. Error detection in data staging introduces three main operations: data computation, data storage, and data movement. Transporting data across compute notes and storing it in DRAM devices increase the energy consumption [12]. Although it seems that adding error detection will increase the energy consumption for the workflow, if we consider that error detection in staging can decrease the total workflow execution time by eliminating substantial computation time in the presence of silent errors, we expect the energy consumption of the workflow to decrease.

\section{RELATED WORK}

Considerable efforts have been directed at developing techniques to detect and remediate silent errors. Process replication or redundancy techniques [18] can achieve a high recall and precision of detection with commensurately high compute and storage cost. Algorithm-based fault tolerance (ABFT) [7] can be very useful in decreasing the error detection cost and are specifically fit for detecting errors in linear algebra kernels using checksums. Detectors based on data analytics have been explored recently to serve as lightweight error detection methods[6, 20]. These approaches use interpolation techniques, such as time series prediction and spatial multivariate interpolation on the scientific dataset. In contrast to these efforts, we provide a framework for in-situ workflows for performing error detection. In addition, any error detection techniques can be easily integrated with our staging based error framework.

Since fail-stop failure is another significant risk for applications and checkpoint/restart has become a standard technique to address it, some research has combined checkpoint/restart with error detection approaches in order to deal with both fail-stop failure and silent errors. The study in [5] provides optimal resilience patterns to cope with fail-stop and silent errors. The research in [10] introduces an on-line ABFT based error detection approach and uses 


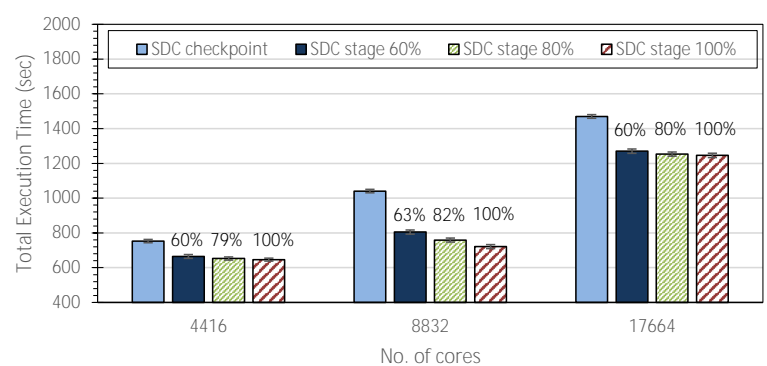

Figure 13: Comparison of the total execution time (in seconds) for the S3D simulation and coupled analysis workflow with error detection in CPU-GPU hybrid staging. Numbers on top of the bars indicate the recall of error detection in staging.

it in conjunction with checkpoint/restart to improve the time required to obtain correct results in an unreliable computing system. These approach are mainly designed for single applications and do not solve the problem of error propagation between coupled application. Our approach specifically targets the coupled scientific simulations and aims to eliminate error propagation via data verification in the shared abstraction, or staging area.

The use of a data staging area has been exploited in several research efforts such as DataStager [2], PreDatA [27], JITStaging [1], DataSpaces [13]/ActiveSpaces [14], and Stacker [23]. Most of these existing data staging solutions focus primarily on fast and asynchronous data movement between components in workflow to lessen the impact of expensive $\mathrm{I} / \mathrm{O}$ operations. In contrast, we present a staging-based error detection framework to fully utilize idle computation capabilities of staging nodes and provide an uniform lightweight error detection service for in-situ workflows.

\section{CONCLUSION AND FUTURE WORK}

Cutting-edge in-situ workflows are generating and consuming data at an ever-growing scale. Silent data corruption is an important issue that needs to be addressed in order to allow these workflows to continue scaling efficiently. In this paper we have presented a staging-based framework for detecting corruption that uses idle computation resource to effectively detect silent errors for in-situ workflows. As an illustrative example, we have demonstrated the use of an improved spatial outlier detection technique to achieve lightweight error detection with high accuracy. We have also provided a CPU-GPU hybrid staging architecture to minimize the impact of error detection on regular I/O operations on a data staging framework.

We have implemented staging based error detection on top of the DataSpaces framework and deployed it on Titan. We have evaluated the effectiveness and performance of our method through accuracy tests, performance tests, synthetic tests and real world large scale S3D application runs. Our experiments demonstrate that staging based error detection framework can effectively reduce the total execution time of scientific workflows when combined with checkpoint/restart. While we evaluated our approach using synthetic tests and the S3D combustion workflow on Titan, as future work we plan to investigate the impact of our approach on other classes of application workflows and machines. We also plan to explore other error detection approaches in staging and evaluate the cost/accuracy trade-offs of these approaches on application workflows.

\section{ACKNOWLEDGMENTS}

This worked was supported by the National Science Foundation (NSF) via grant number CCF-1725649. and by Sandia National Laboratories, a multi-mission laboratory managed and operated by National Technology and Engineering Solutions of Sandia, LLC., a wholly owned subsidiary of Honeywell International, Inc., for the U.S. Department of Energy's National Nuclear Security Administration under contract DE-NA-0003525. This research used resources of the Oak Ridge Leadership Computing Facility at the Oak Ridge National Laboratory, which is supported by the Office of Science of the U.S. Department of Energy under Contract No. DE-AC05-00OR22725. The research at Rutgers was conducted as part of the Rutgers Discovery Informatics Institute $\left(\mathrm{RDI}^{2}\right)$.

We thank Dr. Zheng Zhang from Rutgers University for assistance with CUDA GPU program design, and Dr. Hemanth Kolla from Sandia National Laboratories for help with setup and running of S3D.

\section{REFERENCES}

[1] H. Abbasi, G. Eisenhauer, M. Wolf, K. Schwan, and S. Klasky. Just In Time: Adding Value to The IO Pipelines of High Performance Applications with JITStaging. In Proc. 20th International Symposium on High Performance Distributed Computing (HPDC'11), June 2011.

[2] H. Abbasi, M. Wolf, G. Eisenhauer, S. Klasky, K. Schwan, and F. Zheng. Datastager: scalable data staging services for petascale applications. In Proc. 18th International Symposium on High Performance Distributed Computing (HPDC'09), 2009.

[3] L. Bautista-Gomez and F. Cappello. Detecting silent data corruption for extremescale mpi applications. In Proceedings of the 22nd European MPI Users' Group Meeting (EuroMPI'15), September 2015.

[4] J. Bennett, H. Abbasi, P.-T. Bremer, R. Grout, A. Gyulassy, T. Jin, S. Klasky, H. Kolla, M. Parashar, V. Pascucci, P. Pebay, D. Thompson, H. Yu, F. Zhang, and J. Chen. Combining in-situ and in-transit processing to enable extreme-scale scientific analysis. In High Performance Computing, Networking, Storage and Analysis (SC), 2012 International Conference for, pages 1-9, Nov 2012.

[5] A. Benoit, A. Cavelan, Y. Robert, and H. Sun. Optimal resilience patterns to cope with fail-stop and silent errors. In Proceedings of the 30th IEEE International Parallel and Distributed Processing Symposium (IPDPS'16), May 2016.

[6] E. Berrocal, L. Bautista-Gomez, S. Di, Z. Lan, and F. Cappello. Lightweight silent data corruption detection based on runtime data analysis for hpc applications. In Proc. 24th International Symposium on High Performance Distributed Computing (HPDC'15), June 2015.

[7] G. Bosilca, R. Delmas, J. Dongarra, and J. Langou. Algorithm-based fault tolerance applied to high performance computing. Fournal of Parallel and Distributed Computing, 69(4):410-416, 2009.

[8] F. Cappello, G. Al, W. Gropp, S. Kale, B. Kramer, and M. Snir. Toward exascale resilience: 2014 update. In Supercomputing Frontiers and Innovations: an International fournal, volume 1, pages 5-28, 2014.

[9] J. H. Chen, A. Choudhary, B. de Supinski, M. DeVries, E. R. Hawkes, S. Klasky, W. K. Liao, K. L. Ma, J. Mellor-Crummey, N. Podhorszki, R. Sankaran, S. Shende, and C. S. Yoo. Terascale direct numerical simulations of turbulent combustion using s3d. Computational Science \& Discovery, 2009.

[10] Z. Chen. Online-abft: an online algorithm based fault tolerance scheme for soft error detection in iterative methods. In Proceedings of the 18th ACM SIGPLAN symposium on Principles and practice of parallel programming (PPoPP'13), August 2013.

[11] E. Deelman, T. Peterka, I. Altintas, C. D. Carothers, K. K. van Dam, K. Moreland, M. Parashar, L. Ramakrishnan, M. Taufer, and J. Vetter. The future of scientific workflows. The International fournal of High Performance Computing Applications, 32(1):159-175, 2018.

[12] M. e. M. Diouri, O. Gluck, L. Lefevre, and F. Cappello. Energy considerations in checkpointing and fault tolerance protocols. In Proceedings of the Workshop on IEEE/IFIP International Conference on Dependable Systems and Networks (DSN 2012), June 2012.

[13] C. Docan, M. Parashar, and S. Klasky. Dataspaces: an interaction and coordination framework for coupled simulation workflows. In Proceedings of the 19th ACM International Symposium on High Performance Distributed Computing, HPDC '10, pages 25-36, 2010 . 
[14] C. Docan, F. Zhang, T. Jin, H. Bui, Q. Sun, J. Cummings, N. Podhorszki, S. Klasky, and M. Parashar. Activespaces: Exploring dynamic code deployment for extreme scale data processing. Wiley Online Library, 2014

[15] S. Duan, P. Subedi, K. Teranishi, P. Davis, H. Kolla, M. Gamell, and M. Parashar. Scalable data resilience for in-memory data staging. In Proceedings of the 32th IEEE International Parallel and Distributed Processing Symposium (IPDPS'18), pages 105-115, May 2018

[16] J. Elliott, F. Mueller, M. Stoyanov, and C. G. Webster. Quantifying the impact of single bit flips on floating point arithmetic. In Technical report, Oak Ridge National Laboratory, August 2013.

[17] S. Feng, S. Gupta, A. Ansari, and S. Mahlke. Shoestring: probabilistic soft error reliability on the cheap. In Proceedings of the fifteenth edition of ASPLOS on Architectural support for programming languages and operating systems (ASPLOS'10), pages 385-396, March 2010.

[18] D. Fiala, F. Mueller, C. Engelmann, R. Riesen, K. Ferreira, and R. Brightwell. Detection and correction of silent data corruption for large-scale high-performance computing. In Proceedings of the International Conference on High Performance Computing, Networking, Storage and Analysis (SC'12), November 2012

[19] A. Geist. What is the monster in the closet? In Invited Talk at Workshop on Architectures I: Exascale and Beyond: Gaps in Research, Gaps in our Thinking, volume 2, 2011.

[20] L. B. Gomez and F. Cappello. Detecting silent data corruption through data dynamic monitoring for scientific applications. In the 19th ACM SIGPLAN symposium on Principles and practice of parallel programming (PPoPP), pages 381-382,
February 2014.

[21] M. Parashar. Addressing the petascale data challenge using in-situ analytics. In Proceedings of the 2Nd International Workshop on Petascal Data Analytics: Challenges and Opportunities, PDAC '11, pages 35-36, New York, NY, USA, 2011. ACM.

[22] V. Sridharan, N. DeBardeleben, S. Blanchard, K. B. Ferreira, J. Stearley, J. Shalf, and S. Gurumurthi. Memory errors in modern systems: The good, the bad, and the ugly. In Proceedings of the Twentieth International Conference on Architectural Support for Programming Languages and Operating Systems (ASPLOS'15), March 2015.

[23] P. Subedi, P. Davis, S. Duan, S. Klasky, H. Kolla, and M. Parashar. Stacker: An autonomous data movement engine for extreme-scale data staging-based in-situ workflows. In High Performance Computing, Networking, Storage and Analysis (SC), 2018 International Conference for. IEEE, 2018.

[24] P. Sun and S. Chawla. On local spatial outliers. In Fourth IEEE International Conference on Data Mining, November 2004.

[25] J. T. Daly. A higher order estimate of the optimum checkpoint interval for restart dumps. Future Generation Computer Systems, 3(22):303-312, 2004

[26] U.S. Department of Energy, Office of Science. Exascale computing project. https://www.exascaleproject.org/exascale-computing-project/, 2018.

[27] F. Zheng, H. Abbasi, C. Docan, J. Lofstead, Q. Liu, S. Klasky, M. Parashar, N. Podhorszki, K. Schwan, and M. Wolf. Predata - preparatory data analytics on petascale machines. In Parallel Distributed Processing (IPDPS), 2010 IEEE International Symposium on, pages 1-12, April 2010. 


\section{Appendix: Artifact Description/Artifact Evaluation}

\section{SUMMARY OF THE EXPERIMENTS REPORTED}

We evaluate the staging based error detection framework, implemented as an extension of DataSpaces. In experiments, all staging servers run on the dedicated compute nodes with GPU accelerator on CRAY XK-7.

(1) Performance experiments: run 5 times and calculate average/means as the result, and standard deviation as the error bar.

(2) Synthetic test cases: run 50 times and calculate average/means as the result, and standard deviation as the error bar.

(3) Real-world large scale experiments: run 3 times and calculate average/means as the result, and standard deviation as the error bar.

\section{ARTIFACT AVAILABILITY}

Software Artifact Availability: All author-created software artifacts are maintained in a public repository under an OSI-approved license.

Hardware Artifact Availability: There are no author-created hardware artifacts.

Data Artifact Availability: There are no author-created data artifacts.

Proprietary Artifacts: There are associated proprietary artifacts that are not created by the authors. Some author-created artifacts are proprietary.

List of URLs and/or DOIs where artifacts are available: https://github.com/shaohuaduan/datastaging-error-det 」 $\hookrightarrow$ ection

\section{BASELINE EXPERIMENTAL SETUP, AND MODIFICATIONS MADE FOR THE PAPER}

Relevant hardware details: Cray XK-7

Operating systems and versions: Cray Linux

Compilers and versions: pgi 18.4.0

Applications and versions: S3D 15Oct09, DataSpaces-1.6.1

Libraries and versions: ugni 6.0, cray-mpich 7.6.3

Paper Modifications: We implemented SLOM as an example of staging based error detection framework, integrated it with DataSpaces-1.6.1. tion

Output from scripts that gathers execution environment informa-

CRAY_CUDATOOLKIT_VERSION=9.1.85_3.10-1.0502.df1cc54.।

$\hookrightarrow \quad 3.1$

LESSKEY=/etc/lesskey.bin

MODULE_VERSION_STACK $=3.2 .10 .6$

KSH_AUTOLOAD $=1$

PE_LIBSCI_VOLATILE_PRGENV=CRAY GNU INTEL PGI
PE_SMA_DEFAULT_PKGCONFIG_VARIABLES=PE_SMA_COMPFLAG_@ 」 $\hookrightarrow$ prgenv@

PE_TPSL_64_DEFAULT_GENCOMPS_INTEL_mic_knl=160

MANPATH=/opt/nvidia/cudatoolkit9.1/9.1.85_3.10-1.050

$\hookrightarrow$ 2.df1cc54.3.1/doc/man:/opt/gcc/6.3.0/snos/share/

$\hookrightarrow$ man:/sw/xc30/environment-modules/3.2.10.3/sles11」

$\hookrightarrow$.3_gnu4.9.0/share/man:/sw/sources/hpss/man:/sw/x 」

$\hookrightarrow \mathrm{k6} / \mathrm{man}: / \mathrm{sw} / \mathrm{xk6/hsi/5.0.2.p1/sles11.5/man:/autofs}$

$\hookrightarrow$ /nccs-svm1_sw/titan/.swci/0-login/opt/spack/2017

$\hookrightarrow$ 0612/linux-suse_linux11-x86_64/gcc-5.3.0/git-2.1」

$\hookrightarrow \quad 3.0$-znpqlkovoclvlt5rwm3rkpk7d2m56ez2/share/man:/」

$\hookrightarrow$ sw/xk6/lustredu/1.4/sles11.3_gnu4.8.2/man:/opt/c 」

$\hookrightarrow \mathrm{ray} / \mathrm{atp} / 2.1 .1 / \mathrm{man}$ :/opt/cray/libsci/16.11.1/man:/

$\hookrightarrow$ opt/cray/man/csmlversion:/opt/pgi/18.4.0/linux86」

$\hookrightarrow-64 / 18.4 / \mathrm{man}: / \mathrm{opt} / \mathrm{cray} / \mathrm{mpt} / 7.6 .3 / \mathrm{gni} / \mathrm{man} / \mathrm{mpich}$ :/o

$\hookrightarrow \mathrm{pt} / \mathrm{cray} / \mathrm{craype} / 2.5 .13 / \mathrm{man}: / \mathrm{opt} / \mathrm{cray} / \mathrm{llm} / \mathrm{default} /\rfloor$

$\hookrightarrow \mathrm{man}: /$ opt/cray/lustre-cray_gem_s/2.8.2_3.0.101_0.

$\hookrightarrow$ 46.1_1.0502.8871-1.0502.0.44.1/man:/opt/cray/alp

$\hookrightarrow$ s/5.2.4-2.0502.9950.37.1.gem/man:/opt/modules/3.

$\hookrightarrow$ 2.10.6/share/man:/opt/moab/share/man:/usr/local/」

$\hookrightarrow \mathrm{man}: /$ usr/share/man:/usr/man:/opt/cray/share/man:

$\hookrightarrow$ /opt/cray/man

INFODIR=/usr/local/info:/usr/share/info:/usr/info

NNTPSERVER=news

PE_PAPI_DEFAULT_ACCEL_FAMILY_LIBS_nvidia $=,-1$ cupti,-1 」

$\hookrightarrow$ cudart,-lcuda

PE_PETSC_DEFAULT_GENCOMPILERS_CRAY_sandybridge $=8.6$

PE_PETSC_DEFAULT_GENCOMPS_CRAY_skylake $=86$

PE_TPSL_DEFAULT_GENCOMPS_INTEL_x86_skylake $=160$

PE_CXX_PKGCONFIG_LIBS=mpichcXX

PE_MPICH_GENCOMPILERS_PGI $=15.3$

GNU VERSION $=6.3 .0$

PE_LIBSCI_DEFAULT_GENCOMPS_GNU_mic_knl $=51$

PE_HDF5_DEFAULT_REQUIRED_PRODUCTS=PE_MPICH

HOSTNAME=titan-batch3.ccs.ornl.gov

CRAY_UDREG_INCLUDE_OPTS=-I/opt/cray/udreg/2.3.2-1.05

$\hookrightarrow$ 02.10518.2.17.gem/include

PE_FFTW_DEFAULT_TARGET_mic_knl=mic_knl

PE_LIBSCI_ACC_DEFAULT_PKGCONFIG_VARIABLES=PE_LIBSCI_」

$\hookrightarrow$ ACC_DEFAULT_NV_SUFFIX_@accelerator@

PE_PETSC_DEFAULT_GENCOMPILERS_INTEL_mic_knl=16.0

PE_PETSC_DEFAULT_GENCOMPS_PGI_X86_64=179

PE_TPSL_64_DEFAULT_GENCOMPS_INTEL_interlagos $=160$

PE_TRILINOS_DEFAULT_GENCOMPS_CRAY_X86_64=86

SPACK_ROOT=/ccs/home/USER/codar/spack

PBS_VERSION=TORQUE-6.1.1.1.h2

XKEYSYMDB $=/$ usr $/$ share $/ \mathrm{X} 11 / \mathrm{XKeysymDB}$

CRAY_SITE_LIST_DIR=/etc/opt/cray/modules 
Duan, et al.

LIBRARYMODULES=acml : alps: cray-dwarf: cray-fftw: cray-g 」 $\hookrightarrow$ a:cray-hdf5:cray-hdf5-parallel:cray-libsci:cray-」

$\hookrightarrow$ libsci_acc:cray-mpich:cray-mpich-abi:cray-mpich2」

$\hookrightarrow \quad$ :cray-netcdf:cray-netcdf-hdf5parallel:cray-paral」

$\hookrightarrow$ lel-netcdf:cray-petsc:cray-petsc-complex:cray-sh」

$\hookrightarrow$ mem:cray-tpsl:cray-trilinos:cudatoolkit:fftw:ga:」

$\hookrightarrow$ hdf5:hdf5-parallel:iobuf:libfast:netcdf:netcdf-h」

$\hookrightarrow \quad$ df5parallel:ntk:onesided:papi:petsc:petsc-comple

$\hookrightarrow \quad \mathrm{x}:$ pmi:tpsl:trilinos:xt-libsci:xt-mpich2:xt-mpt: $\mathrm{x}_{\rfloor}$

$\hookrightarrow \quad \mathrm{t}$-papi

RCLOCAL_BASEOPTS=true

PE_ACML_DEFAULT_DIR_GNU=gfortran64

PE_NETCDF_DEFAULT_VOLATILE_PKGCONFIG_PATH=/opt/cray/ 」

$\hookrightarrow$ netcdf/4.4.1.1.3/@PRGENV@/@PE_NETCDF_DEFAULT_GEN 」

$\hookrightarrow$ COMPSe/lib/pkgconfig

PE_PARALLEL_NETCDF_DEFAULT_VOLATILE_PKGCONFIG_PATH=/ ।

$\hookrightarrow$ opt/cray/parallel-netcdf/1.8.1.3/@PRGENVQ/@PE_PA」

$\hookrightarrow$ RALLEL_NETCDF_DEFAULT_GENCOMPS@/lib/pkgconfig

PE_SMA_DEFAULT_COMPFLAG_GNU=-fcray-pointer

PE_TPSL_64_DEFAULT_GENCOMPS_PGI_X86_64=153

PE_TRILINOS_DEFAULT_VOLATILE_PKGCONFIG_PATH=/opt/cra 」

$\hookrightarrow y / t r i l i n o s / 12.10 .1 .1 /$ /QPRGENVQ/@PE_TRILINOS_DEFAU 」

$\hookrightarrow$ LT_GENCOMPS@/@PE_TRILINOS_DEFAULT_TARGET@/lib/pk」

$\hookrightarrow$ gconfig

PE_ACML_DEFAULT_DIR_DEFAULT64=_int64

$P E \_E N V=P G I$

PE_HDF5_DEFAULT_GENCOMPILERS_GNU $=5.14 .9$

PE_MPICH_ALTERNATE_LIBS_dpm=_dpm

PE_SMA_DEFAULT_COMPFLAG=

PE_TPSL_64_DEFAULT_GENCOMPILERS_CRAY_X86_64=8.6

PBS_ACCOUNT=CSC143

SHELL=/bin/bash

HOST=t i tan-batch3

PE_ACML_DEFAULT_TARGET_interlagos $=$ _fma4

PE_TPSL_DEFAULT_GENCOMPS_CRAY_x86_skylake $=86$

PKGCONFIG_ENABLED $=1$

HISTSIZE $=1000$

PROFILEREAD=true

PE_PETSC_DEFAULT_GENCOMPS_CRAY_sandybridge $=86$

PE_TPSL_DEFAULT_GENCOMPILERS_GNU_X86_skylake $=6.1$

XTOS_VERSION $=5.2 .82$

PBS_JOBNAME $=$ test_dspaces_titan

CRAY_UGNI_POST_LINK_OPTS=-L/opt/cray/ugni / 6.0-1.0502 」

$\hookrightarrow \quad$ 10863.8.28.gem/lib64

CRAY_XPMEM_POST_LINK_OPTS=-L/opt/cray/xpmem/0.1-2.05」

$\hookrightarrow \quad 02.64982 .7 .26 . \mathrm{gem} / \mathrm{lib64}$

CRAYPE_DIR=/opt/cray/craype/2.5.13

PE_NETCDF_DEFAULT_VOLATILE_PRGENV=GNU

PE_PARALLEL_NETCDF_DEFAULT_VOLATILE_PRGENV=GNU

PE_PETSC_DEFAULT_GENCOMPS_GNU_haswell=53 49

PE_PETSC_DEFAULT_GENCOMPS_INTEL_haswell $=160$

PE_PETSC_DEFAULT_GENCOMPS_PGI_interlagos $=179$

PE_TPSL_64_DEFAULT_GENCOMPS_INTEL_x86_skylake $=160$

PE_TPSL_DEFAULT_GENCOMPS_GNU_sandybridge $=5149$

PE_TPSL_DEFAULT_REQUIRED_PRODUCTS=PE_MPICH:PE_LIBSCI

PE_TRILINOS_DEFAULT_VOLATILE_PRGENV=CRAY GNU INTEL
PE_MPICH_DIR_PGI_DEFAULT64 $=64$

OLCF_MODULATOR=/sw/sources/modulator/1.2.0

TMPDIR $=/ \mathrm{tmp} / \mathrm{scratch}$

PERL5LIB=/opt/moab/lib/perl5

PE_ACML_DEFAULT_DIR_CRAY=gfortran 64

PE_FFTW_DEFAULT_VOLATILE_PKGCONFIG_PATH=/opt/cray/ff 」

$\hookrightarrow$ tw/3.3.6.2/@PE_FFTW_DEFAULT_TARGETa/lib/pkgconfig

PE_HDF5_DEFAULT_VOLATILE_PRGENV=GNU

PE_HDF5_PARALLEL_DEFAULT_VOLATILE_PKGCONFIG_PATH=/op 」

$\hookrightarrow \quad \mathrm{t} / \mathrm{cray} / \mathrm{hdf5}$-parallel/1.10.0.3/@PRGENV@/@PE_HDF5_

$\hookrightarrow$ PARALLEL_DEFAULT_GENCOMPS@/lib/pkgconfig

PE_LIBSCI_DEFAULT_GENCOMPILERS_CRAY_mic_knl=8.5

PE_LIBSCI_GENCOMPS_CRAY_mic_knl $=85$

PE_NETCDF_HDF5PARALLEL_DEFAULT_VOLATILE_PKGCONFIG_PA 」

$\hookrightarrow \mathrm{TH}=/ \mathrm{opt} / \mathrm{cray} / \mathrm{netcdf}$-hdf5parallel/4.4.1.1.3/@PRGE 」

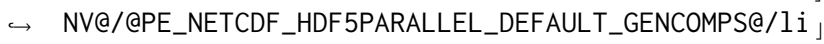

$\hookrightarrow$ b/pkgconfig

PE_PETSC_DEFAULT_GENCOMPILERS_PGI_x86_64=17.9

PE_PETSC_DEFAULT_GENCOMPS_CRAY_interlagos $=86$

PE_TPSL_64_DEFAULT_GENCOMPILERS_PGI_interlagos $=15.3$

CRAY_MPICH2_DIR=/opt/cray/mpt/7.6.3/gni/mpich-pgi/15」

$\hookrightarrow \quad .3$

ALT_LINKER=/sw/xk6/xalt $/ 0.7 .5 / \mathrm{bin} / \mathrm{ld}$

LIBRARY_PATH=/autofs/nccs-svm1_sw/titan/.swci/0-logi 」

$\hookrightarrow$ n/opt/spack/20170612/linux-suse_linux11-x86_64/g 」

$\hookrightarrow$ cc-5.3.0/git-2.13.0-znpqlkovoclvlt5rwm3rkpk7d2m5」

$\hookrightarrow 6 \mathrm{ez} 2 / 1 \mathrm{ib}$

XALT_DIR=/sw/xk6/xalt/0.7.5

CRAY_CUDATOOLKIT_POST_LINK_OPTS=-L/opt/nvidia/cudato 」

$\hookrightarrow$ olkit9.1/9.1.85_3.10-1.0502.df1cc54.3.1/1ib64

$\hookrightarrow \quad$-L/opt/nvidia/cudatoolkit9.1/9.1.85_3.10-1.0502.

$\hookrightarrow$ df1cc54.3.1/extras/CUPTI/lib64 -W1,--as-needed

$\hookrightarrow \quad-W 1,-1$ cupti -Wl,-lcudart -Wl,--no-as-needed

$\hookrightarrow \quad-$ L/opt/cray/nvidia/default/lib64 -lcuda

PE_GA_DEFAULT_VOLATILE_PRGENV=GNU

PE_LIBSCI_DEFAULT_GENCOMPS_GNU_X86_64=61 5149

PE_TPSL_64_DEFAULT_GENCOMPILERS_CRAY_interlagos $=8.6$

PE_TPSL_DEFAULT_GENCOMPS_CRAY_mic_knl $=86$

CRAY_PRGENVPGI $=$ loaded

PBS_ENVIRONMENT=PBS_BATCH

MORE $=-s 1$

FPATH=:/opt/modules/3.2.10.6/init/sh_funcs/no_redire 」

$\hookrightarrow \mathrm{ct}$ :/opt/modules/3.2.10.6/init/sh_funcs/no_redire

$\hookrightarrow \quad c t$

PE_LIBSCI_ACC_DEFAULT_GENCOMPS_CRAY_x86_64=85

PE_LIBSCI_ACC_DEFAULT_REQUIRED_PRODUCTS=PE_MPICH:PE_

$\hookrightarrow \quad$ LIBSCI

PE_LIBSCI_GENCOMPILERS_CRAY_mic_knl $=8.5$

PE_MPICH_DEFAULT_GENCOMPILERS_GNU=5.1 4.9

PE_PKGCONFIG_PRODUCTS=PE_LIBSCI : PE_MPICH

PE_TPSL_DEFAULT_GENCOMPS_INTEL_X86_64=160

PE_MPICH_GENCOMPS_GNU $=5149$

QTDIR $=/$ usr $/$ lib/qt3

PE_PAPI_DEFAULT_ACCEL_LIBS_nvidia35=,-lcupti ,-lcudar 」

$\hookrightarrow \quad t,-$ lcuda 
PE_PETSC_DEFAULT_REQUIRED_PRODUCTS=PE_MPICH:PE_LIBSC 」

$\hookrightarrow \quad I: P E \_H D F 5$ PARALLEL:PE_TPSL

PE_TPSL_64_DEFAULT_GENCOMPS_CRAY_haswell=86

PE_TPSL_64_DEFAULT_VOLATILE_PKGCONFIG_PATH=/opt/cray ।

$\hookrightarrow /$ tpsl/17.11.1/@PRGENV@64/@PE_TPSL_64_DEFAULT_GEN 」

$\hookrightarrow \quad$ COMPS@/@PE_TPSL_64_DEFAULT_TARGET@/lib/pkgconfig

PE_CRAY_DEFAULT_FIXED_PKGCONFIG_PATH=/opt/cray/paral 」

$\hookrightarrow$ lel-netcdf/1.8.1.3/CRAY/8.6/lib/pkgconfig:/opt/c 」

$\hookrightarrow \mathrm{ray} / \mathrm{netcdf}$-hdf5parallel/4.4.1.1.3/CRAY/8.6/lib/p」

$\hookrightarrow$ kgconfig:/opt/cray/netcdf/4.4.1.1.3/CRAY/8.6/lib」

$\hookrightarrow$ /pkgconfig:/opt/cray/hdf5-parallel/1.10.0.3/CRAY」

$\hookrightarrow$ /8.6/lib/pkgconfig:/opt/cray/hdf5/1.10.0.3/CRAY/」

$\hookrightarrow$ 8.6/lib/pkgconfig:/opt/cray/ga/5.3.0.7/CRAY/8.4/」

$\hookrightarrow \quad \mathrm{ib} /$ pkgconfig

PE_TRILINOS_DEFAULT_GENCOMPILERS_CRAY_X86_64=8.6

PBS_0_WORKDIR=/lustre/atlas2/csc143/scratch/USER/dua 」

$\hookrightarrow$ nspaper

PE_LIBSCI_DEFAULT_OMP_REQUIRES_openmp=_mp

PE_PETSC_DEFAULT_GENCOMPILERS_PGI_interlagos $=17.9$

PE_PETSC_DEFAULT_GENCOMPS_CRAY_X86_64=86

PE_TPSL_64_DEFAULT_GENCOMPILERS_CRAY_sandybridge $=8.6$

PE_TPSL_64_DEFAULT_GENCOMPILERS_PGI_X86_64=15.3

PE_FORTRAN_PKGCONFIG_LIBS $=$ mpich 90

PE_SMA_DEFAULT_VOLATILE_PKGCONFIG_PATH=/opt $/ \mathrm{cray} / \mathrm{mpt}$ 」

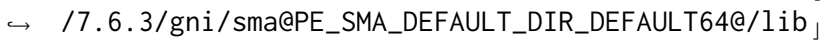

$\hookrightarrow \quad 64 /$ pkgconfig

ALLINEA_QUEUE_DLL=/opt/cray/mpt/7.6.3/gni/mpich-pgi/」

$\hookrightarrow \quad 15.3 / 1$ ib/libtvmpich.so.3.0.1

LC_ALL=POSIX

ALPS_APP_ID $=20266923$

PE_ACML_DEFAULT_DIR_INTEL=i for $t 64$

PE_LIBSCI_ACC_DEFAULT_VOLATILE_PRGENV=CRAY GNU

PE_TRILINOS_DEFAULT_GENCOMPS_INTEL_X86_64=160

CRAY_MPICH_BASEDIR=/opt/cray/mpt/7.6.3/gni

USER=USER

PBS_TASKNUM $=1$

JRE_HOME=/usr/lib64/jvm/java/jre

PE_ACML_DEFAULT_TARGET_x86_64=

PE_HDF5_PARALLEL_DEFAULT_GENCOMPILERS_GNU=5.1 4.9

PE_NETCDF_HDF5PARALLEL_DEFAULT_GENCOMPILERS_GNU=5.1

$\hookrightarrow \quad 4.9$

PE_TPSL_64_DEFAULT_GENCOMPS_CRAY_x86_skylake $=86$

PE_TPSL_64_DEFAULT_GENCOMPS_INTEL_haswell $=160$

CRAYPE_LINK_TYPE=dynamic

LS_COLORS=

PE_FFTW_DEFAULT_TARGET_interlagos=interlagos

PE_LIBSCI_DEFAULT_VOLATILE_PRGENV=CRAY GNU INTEL PGI

PE_LIBSCI_GENCOMPILERS_GNU_mic_knl=5.1

PE_PETSC_DEFAULT_GENCOMPILERS_INTEL_interlagos $=16.0$

PE_TPSL_64_DEFAULT_GENCOMPILERS_INTEL_mic_knl $=16.0$

PE_TPSL_DEFAULT_GENCOMPS_CRAY_X86_64=86

PE_TRILINOS_DEFAULT_GENCOMPILERS_GNU_X86_64=5. 14.9

PE_TRILINOS_DEFAULT_GENCOMPILERS_INTEL_X86_64=16.0
LD_LIBRARY_PATH=/opt/gcc/6.3.0/snos/lib64:/autofs/nc 」

$\hookrightarrow$ cs-svm1_sw/titan/.swci/0-login/opt/spack/2017061」

$\hookrightarrow$ 2/linux-suse_linux11-x86_64/gcc-5.3.0/git-2.13.0」

$\hookrightarrow$-znpqlkovoclvlt5rwm3rkpk7d2m56ez2/lib: /opt/pgi/18」

$\hookrightarrow .4 .0 / 1$ inux86-64/18.4/lib

PBS_O_HOME $=/ \mathrm{ccS} / \mathrm{home} /$ USER

PE_LIBSCI_PKGCONFIG_VARIABLES=PE_LIBSCI_OMP_REQUIRES 」

$\hookrightarrow$ _@openmp@

PE_PETSC_DEFAULT_VOLATILE_PRGENV=CRAY CRAY64 GNU

$\hookrightarrow \quad$ GNU64 INTEL INTEL64 PGI PGI64

PE_PKGCONFIG_LIBS=cray-cudatoolkit:AtpSigHandler: $1 \mathrm{ib}$

$\hookrightarrow$ sci_mpi:libsci:mpich

PE_TPSL_64_DEFAULT_GENCOMPILERS_GNU_sandybridge $=5.1$

$\hookrightarrow \quad 4.9$

PE_TPSL_64_DEFAULT_GENCOMPILERS_INTEL_haswell=16.0

PE_MPICH_FIXED_PRGENV=INTEL

XNLSPATH=/usr/share/X11/nls

PE_PETSC_DEFAULT_GENCOMPILERS_CRAY_mic_knl=8.6

PE_PETSC_DEFAULT_GENCOMPILERS_CRAY_X86_64=8.6

PE_PETSC_DEFAULT_GENCOMPILERS_INTEL_skylake $=16.0$

PE_PETSC_DEFAULT_GENCOMPS_GNU_interlagos $=5349$

PE_PETSC_DEFAULT_GENCOMPS_GNU_sandybridge $=5349$

PE_PETSC_DEFAULT_GENCOMPS_INTEL_interlagos $=160$

PE_PETSC_DEFAULT_GENCOMPS_INTEL_sandybridge $=160$

PE_TPSL_DEFAULT_GENCOMPS_GNU_haswell=51 49

WORKDIR $=/ \mathrm{tmp} /$ work/USER

$\mathrm{ENV}=/$ etc/bash. bashrc

MPICH_ABORT_ON_ERROR=1

PE_LIBSCI_DEFAULT_GENCOMPS_CRAY_X86_64=83

PE_PAPI_DEFAULT_PKGCONFIG_VARIABLES=PE_PAPI_ACCEL_LI 」

$\hookrightarrow$ BS_Qaccelerator@

PE_PETSC_DEFAULT_GENCOMPILERS_CRAY_haswell=8.6

PE_PETSC_DEFAULT_GENCOMPS_GNU_mic_knl $=53$

PE_PETSC_DEFAULT_GENCOMPS_INTEL_mic_knl $=160$

PE_TPSL_64_DEFAULT_GENCOMPILERS_GNU_interlagos $=5.1$

$\hookrightarrow \quad 4.9$

PE_TPSL_64_DEFAULT_GENCOMPS_INTEL_sandybridge $=160$

MPICH_DIR=/opt/cray/mpt/7.6.3/gni/mpich-pgi/15.3

PGI_VERS_STR $=18.4 .0$

HOSTTYPE $=x 86 \_64$

ATP_POST_LINK_OPTS=-W1, -L/opt/cray/atp/2.1.1/1ibApp/

PE_FFTW_DEFAULT_REQUIRED_PRODUCTS=PE_MPICH

PE_FFTW_DEFAULT_TARGET_sandybridge=sandybridge

PE_HDF5_PARALLEL_DEFAULT_REQUIRED_PRODUCTS=PE_MPICH

PE_NETCDF_HDF5PARALLEL_DEFAULT_REQUIRED_PRODUCTS=PE_ 」

$\hookrightarrow \quad$ HDF5_PARALLEL:PE_MPICH

PE_PETSC_DEFAULT_GENCOMPILERS_INTEL_sandybridge $=16.0$

PE_TPSL_64_DEFAULT_GENCOMPILERS_CRAY_haswell=8.6

PE_MPICH_FORTRAN_PKGCONFIG_LIBS $=$ mpi $\operatorname{chf} 90$

PBS_WALLTIME $=300$

PE_PETSC_DEFAULT_GENCOMPILERS_GNU_mic_knl=5.3

RCLOCAL_PRGENV=true

PBS_MOMPORT $=15003$

PBS_GPUFILE=/var/spool/torque/aux//4642066gpu

FROM_HEADER= 
Duan, et al.

PE_PRODUCT_LIST =CRAY_PMI : CRAY_LIBSCI : PGI : CRAYPE : CRAY」 $\hookrightarrow$ PE_INTERLAGOS:CRAY_LLM:CRAY_XPMEM:CRAY_DMAPP:CRA」 $\hookrightarrow \quad$ Y_UGNI:CRAY_UDREG:CRAY_ALPS PE_LIBSCI_DEFAULT_GENCOMPILERS_INTEL_X86_64=15.0 PE_LIBSCI_GENCOMPS_INTEL_X86_64 $=150$ PE_TPSL_DEFAULT_GENCOMPILERS_CRAY_X86_64=8.6 PE_TPSL_DEFAULT_GENCOMPS_GNU_interlagos $=5149$ GCC_VERSION $=6.3 .0$

PAGER=less

PE_MPICH_DEFAULT_GENCOMPS_PGI $=153$

PE_PETSC_DEFAULT_GENCOMPILERS_GNU_X86_64=5.3 4.9

PE_TPSL_DEFAULT_GENCOMPS_GNU_x86_skylake $=61$

CRAY_MPICH_ROOTDIR=/opt/cray/mpt/7.6.3

PGI_VERSION $=18.4$

ALPS_APP_PE $=0$

CSHEDIT=emacS

PE_LIBSCI_GENCOMPILERS_GNU_X86_64=6.1 5.14 .9

PE_PETSC_DEFAULT_GENCOMPS_GNU_skylake $=61$

PE_PETSC_DEFAULT_GENCOMPS_INTEL_skylake $=160$

PE_TPSL_64_DEFAULT_GENCOMPILERS_INTEL_X86_64=16.0

PE_MPICH_GENCOMPILERS_CRAY $=8.6$

PE_MPICH_MODULE_NAME $=$ cray-mpich

PROJWORK=/lustre/atlas/proj-shared

SPS_HOME=/ccs/home/USER/astro/fsps

PBS_O_QUEUE=debug

XDG_CONFIG_DIRS $=/$ etc $/ x d g$

PE_LIBSCI_DEFAULT_GENCOMPILERS_CRAY_X86_64=8.3

PE_LIBSCI_GENCOMPS_CRAY_X86_64=83

PE_MPICH_DEFAULT_VOLATILE_PRGENV=CRAY GNU PGI

PE_MPICH_TARGET_VAR_nvidia2 $0=-1$ cudart

PE_TPSL_64_DEFAULT_REQUIRED_PRODUCTS=PE_MPICH:PE_LIB 」

$\hookrightarrow$ SCI

PE_TPSL_DEFAULT_GENCOMPS_CRAY_haswell=86

PE_TPSL_DEFAULT_GENCOMPS_CRAY_sandybridge $=86$

MINICOM $=-C$ on

USERMODULES=PrgEnv-cray:PrgEnv-gnu:PrgEnv-intel:PrgE 」

$\hookrightarrow$ nv-pathscale:PrgEnv-pgi:acml:alps:apprentice:app 」

$\hookrightarrow$ rentice 2 :atp:blcr:cce:chapel:cray-ccdb:cray-fftw

$\hookrightarrow \quad$ :cray-ga:cray-hdf5:cray-hdf5-parallel:cray-lgdb: 」

$\hookrightarrow$ cray-libsci:cray-libsci_acc:cray-mpich:cray-mpic」

$\hookrightarrow$ h-compat:cray-mpich2:cray-netcdf:cray-netcdf-hdf J

$\hookrightarrow$ 5parallel:cray-parallel-netcdf:cray-petsc:cray-p」

$\hookrightarrow$ etsc-complex:cray-shmem:cray-snplauncher:cray-tp」

$\hookrightarrow$ sl:cray-trilinos:craypat:craype:craypkg-gen:cuda」

$\hookrightarrow$ toolkit:ddt:fftw:ga:gcc:hdf5:hdf5-parallel:intel」

$\hookrightarrow \quad$ :iobuf: java:lgdb:libfast:libsci_acc:mpich1:netcd

$\hookrightarrow \quad f:$ netcdf-hdf5parallel:netcdf-nofsync:netcdf-nofs J

$\hookrightarrow$ ync-hdf5parallel:ntk:onesided:papi:parallel-netc」

$\hookrightarrow$ df:pathscale:perftools:perftools-lite:petsc:pets」

$\hookrightarrow \quad$ c-complex:pgi:pmi:stat:totalview:tpsl:trilinos: $x_{\text {J }}$

$\hookrightarrow$ t-asyncpe: xt-craypat:xt-lgdb:xt-libsci:xt-mpich2 」

$\hookrightarrow \quad$ :xt-mpt:xt-papi:xt-shmem:xt-totalview

CRAY_DMAPP_INCLUDE_OPTS=-I/opt/cray/dmapp/7.0.1-1.05 」

$\hookrightarrow \quad 02.11080 .8 .74$. gem/include

$\hookrightarrow \quad-$ I/opt/cray/gni-headers/4.0-1.0502.10859.7.8.gem 」

$\hookrightarrow$ /include
CRAY_LIBSCI_BASE_DIR=/opt/cray/libsci/16.11.1

CRAY_LIBSCI_DIR=/opt/cray/libsci/16.11.1

PE_ACML_DEFAULT_DIR_PGI=pgi64

PE_LIBSCI_ACC_DEFAULT_VOLATILE_PKGCONFIG_PATH=/opt/c 」

$\hookrightarrow \mathrm{ray} / \mathrm{libsci}$ acc/18.04.1/@PRGENV@/@PE_LIBSCI_ACC_D 」

$\hookrightarrow$ EFAULT_GENCOMPSQ/@PE_LIBSCI_ACC_DEFAULT_TARGET@/ ।

$\hookrightarrow \quad \mathrm{ib} /$ pkgconfig

PE_LIBSCI_PKGCONFIG_LIBS=libsci_mpi : libsci

PE_NETCDF_DEFAULT_GENCOMPS_GNU=51 49

PE_PARALLEL_NETCDF_DEFAULT_GENCOMPS_GNU=51 49

PE_TPSL_64_DEFAULT_GENCOMPS_GNU_mic_knl $=51$

PE_TPSL_64_DEFAULT_GENCOMPS_GNU_X86_64=51 49

$\mathrm{PGI}=/$ opt/pgi/18.4.0

PBS_O_LOGNAME=USER

$\mathrm{PATH}=/$ opt/nvidia/cudatoolkit9.1/9.1.85_3.10-1.0502.d

$\hookrightarrow$ f1cc54.3.1/bin:/opt/nvidia/cudatoolkit9.1/9.1.85

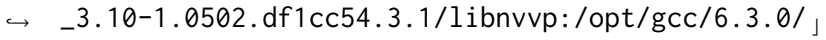

$\hookrightarrow$ bin:/ccs/home/USER/codar/spack/bin:/sw/xk6/bin:/」

$\hookrightarrow$ sw/xk6/hsi/5.0.2.p1/sles11.5/bin:/autofs/nccs-sv」

$\hookrightarrow$ m1_sw/titan/.swci/0-login/opt/spack/20170612/lin」

$\hookrightarrow$ ux-suse_linux11-x86_64/gcc-5.3.0/git-2.13.0-znpq

$\hookrightarrow \quad$ lkovoclvlt5rwm3rkpk7d2m56ez2/bin:/sw/xk6/xalt/0.」

$\hookrightarrow$ 7.5/bin:/sw/xk6/lustredu/1.4/sles11.3_gnu4.8.2/b」

$\hookrightarrow \quad$ in:/opt/cray/pmi/5.0.9-1.0000.10911.175.4.gem/bi 」

$\hookrightarrow \mathrm{n}: / \mathrm{opt} / \mathrm{pgi} / 18.4 .0 /$ linux86-64/18.4/bin:/opt/cray/」

$\hookrightarrow \mathrm{mpt} / 7.6 .3 / \mathrm{gni} / \mathrm{bin}$ /opt/cray/craype/2.5.13/bin:/o 」

$\hookrightarrow \mathrm{pt} / \mathrm{cray} / \mathrm{llm} / \mathrm{default} / \mathrm{bin}$ : /opt/cray/llm/default/et 」

$\hookrightarrow \mathrm{c}: /$ opt/cray/xpmem/0.1-2.0502.64982.7.26.gem/bin: 」

$\hookrightarrow$ /opt/cray/ugni/6.0-1.0502.10863.8.28.gem/bin:/op」

$\hookrightarrow \quad t / c r a y / u d r e g / 2.3 .2-1.0502 .10518 .2 .17 . g e m / b i n: / o p 」$

$\hookrightarrow \quad t / c r a y / l u s t r e-c r a y \_g e m \_s / 2.8 .2 \_3.0 .101 \_0.46 .1 \_1$.」

$\hookrightarrow$ 0502.8871-1.0502.0.44.1/sbin:/opt/cray/lustre-cr 」

$\hookrightarrow \quad$ ay_gem_s/2.8.2_3.0.101_0.46.1_1.0502.8871-1.0502」

$\hookrightarrow \quad .0 .44 .1 / \mathrm{bin}: / o p t / c r a y / a l p s / 5.2 .4-2.0502 .9950 .37$.

$\hookrightarrow$ 1.gem/sbin:/opt/cray/alps/5.2.4-2.0502.9950.37.1」

$\hookrightarrow$.gem/bin:/opt/cray/sdb/1.1-1.0502.63652.4.27.gem」

$\hookrightarrow$ /bin:/opt/cray/nodestat/2.2-1.0502.60539.1.31.ge

$\hookrightarrow \mathrm{m} / \mathrm{bin}$ :/opt/modules/3.2.10.6/bin:/usr/bin:/usr/sb」

$\hookrightarrow$ in:/opt/moab/bin:/ccs/home/USER/bin:/usr/local/b」

$\hookrightarrow$ in:/usr/bin:/bin:/usr/bin/X11:/usr/X11R6/bin:/us」

$\hookrightarrow$ r/games:/opt/bin:/usr/lib/mit/bin:/usr/lib/mit/s」

$\hookrightarrow$ bin:/usr/lib/qt3/bin:/opt/cray/bin:/opt/bin:/opt 」

$\hookrightarrow$ /public/bin:/opt/bin:/opt/public/bin:/cm/local/a」

$\hookrightarrow$ pps/environment-modules/3.2.10/bin

MAIL=/var/spool/mail/USER

MODULE_VERSION $=3.2 .10 .6$

PE_HDF5_DEFAULT_VOLATILE_PKGCONFIG_PATH=/opt/cray/hd ।

$\hookrightarrow \quad \mathrm{f5} / 1.10 .0 .3$ /@PRGENVQ/@PE_HDF5_DEFAULT_GENCOMPS@/ ।

$\hookrightarrow \quad \mathrm{ib} / \mathrm{pkgconfig}$

PE_PKGCONFIG_DEFAULT_PRODUCTS=PE_TRILINOS:PE_TPSL_64 」

$\hookrightarrow \quad: P E \_T P S L: P E \_P E T S C: P E \_P A R A L L E L \_N E T C D F: P E \_N E T C D F \_H$ 」

$\hookrightarrow$ DF5PARALLEL:PE_NETCDF:PE_MPICH:PE_LIBSCI_ACC:PE_」

$\hookrightarrow \quad$ LIBSCI:PE_HDF5_PARALLEL:PE_HDF5:PE_GA:PE_FFTW2:P」

$\hookrightarrow$ E_FFTW:PE_ACML

PE_TPSL_DEFAULT_GENCOMPILERS_GNU_X86_64=5. 14.9

PE_TPSL_DEFAULT_GENCOMPILERS_PGI_X86_64=15.3 
PE_TPSL_DEFAULT_GENCOMPS_CRAY_interlagos $=86$

PE_MPICH_GENCOMPILERS_GNU=5.1 4.9

APRUN_XFER_LIMITS $=0$

ALPS_APP_DEPTH=1

PBS_0_LANG=en_US.UTF-8

$\mathrm{CPU}=\mathrm{x} 86$ 6 64

XTPE_NETWORK_TARGET=gemini

ATP_IGNORE_SIGTERM $=1$

PE_FFTW_DEFAULT_TARGET_abudhabi=abudhabi

PE_LIBSCI_DEFAULT_GENCOMPS_PGI_x86_64=153

PE_MPICH_DEFAULT_DIR_PGI_DEFAULT64 64

PE_NETCDF_DEFAULT_GENCOMPILERS_GNU=5.1 4.9

PE_PARALLEL_NETCDF_DEFAULT_GENCOMPILERS_GNU=5.1 4.9

PE_PETSC_DEFAULT_GENCOMPS_CRAY_mic_knl $=86$

PE_TPSL_64_DEFAULT_GENCOMPILERS_GNU_x86_skylake $=6.1$

PE_TPSL_DEFAULT_GENCOMPILERS_GNU_haswell=5.1 4.9

_=/usr/bin/env

PBS_JOBC00KIE=6C6A7D68D1D84BD6A3DBD487ECB61906

JAVA_BINDIR=/usr/lib64/jvm/java/bin

PE_HDF5_PARALLEL_DEFAULT_FIXED_PRGENV=CRAY PGI INTEL

PE_HDF5_PARALLEL_DEFAULT_GENCOMPS_GNU $=5149$

PE_NETCDF_HDF5PARALLEL_DEFAULT_FIXED_PRGENV=CRAY PGI

$\hookrightarrow \quad$ INTEL

PE_NETCDF_HDF5PARALLEL_DEFAULT_GENCOMPS_GNU $=5149$

PE_SMA_DEFAULT_DIR_CRAY_DEFAULT T4 $=64$

PE_TPSL_64_DEFAULT_GENCOMPILERS_CRAY_x86_skylake $=8.6$

CRAY_UDREG_POST_LINK_OPTS=-L/opt/cray/udreg/2.3.2-1 . 」

$\hookrightarrow$ 0502.10518.2.17.gem/lib64

PE_LIBSCI_DEFAULT_GENCOMPILERS_INTEL_mic_knl=15.0

PE_LIBSCI_GENCOMPS_INTEL_mic_knl $=150$

PE_TPSL_64_DEFAULT_GENCOMPS_CRAY_sandybridge $=86$

PE_TPSL_64_DEFAULT_VOLATILE_PRGENV=CRAY CRAY64 GNU

$\hookrightarrow \quad$ GNU64 INTEL INTEL64 PGI PGI64

PE_TPSL_DEFAULT_GENCOMPILERS_CRAY_mic_knl $=8.6$

PE_TPSL_DEFAULT_GENCOMPS_INTEL_interlagos $=160$

$\mathrm{PWD}=/$ lustre/atlas/scratch/USER/csc143/duanspaper

INPUTRC $=/$ etc $/$ inputrc

CRAY_ALPS_POST_LINK_OPTS=-L/opt/cray/alps/5.2.4-2.05 」

$\hookrightarrow \quad 02.9950 .37 .1 . \mathrm{gem} / \mathrm{lib64}$

CRAYPE_VERSION=2.5.13

PE_TPSL_DEFAULT_GENCOMPS_GNU_mic_knl $=51$

PE_MPICH_VOLATILE_PRGENV=CRAY GNU PGI

JAVA_HOME=/usr/lib64/jvm/java
TARGETMODULES=craype-abudhabi : craype-abudhabi-cu: $c r a 」$

$\hookrightarrow$ ype-accel-host:craype-accel-nvidia20:craype-acce

$\hookrightarrow$ 1-nvidia30:craype-accel-nvidia35:craype-barcelon

$\hookrightarrow$ a:craype-broadwell:craype-haswell:craype-hugepag 」

$\hookrightarrow$ es128K: craype-hugepages 128M:craype-hugepages 16M: 」

$\hookrightarrow$ craype-hugepages256M: craype-hugepages $2 \mathrm{M}:$ craype-h」

$\hookrightarrow$ ugepages $32 \mathrm{M}$ :craype-hugepages4M:craype-hugepages 5

$\hookrightarrow \quad 12 \mathrm{~K}:$ craype-hugepages 512M:craype-hugepages64M:cra

$\hookrightarrow$ ype-hugepages8M:craype-intel-knc:craype-interlag

$\hookrightarrow \quad$ os:craype-interlagos-cu:craype-istanbul:craype-i

$\hookrightarrow \quad$ vybridge:craype-mc12:craype-mc8:craype-mic-knl:c」

$\hookrightarrow$ raype-network-aries:craype-network-gemini:craype

$\hookrightarrow$-network-infiniband:craype-network-none: craype-ne

$\hookrightarrow$ twork-seastar:craype-sandybridge:craype-shanghai 」

$\hookrightarrow$ :craype-target-compute_node:craype-target-local_」

$\hookrightarrow$ host:craype-target-native:craype-xeon:xtpe-barce

$\hookrightarrow$ lona:xtpe-interlagos:xtpe-interlagos-cu:xtpe-ist

$\hookrightarrow \quad$ anbul:xtpe-mc12:xtpe-mc8:xtpe-network-gemini:xtp

$\hookrightarrow$ e-network-seastar:xtpe-shanghai:xtpe-target-nati

$\hookrightarrow \quad$ ve:xtpe-xeon

_LMFILES_=/opt/modulefiles/modules/3.2.10.6: /opt/cra 」

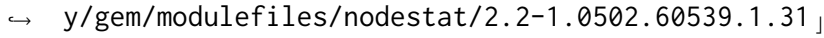

$\hookrightarrow$.gem:/opt/cray/gem/modulefiles/sdb/1.1-1.0502.63।

$\hookrightarrow$ 652.4.27.gem:/opt/cray/gem/modulefiles/alps/5.2.」

$\hookrightarrow$ 4-2.0502.9950.37.1.gem:/opt/cray/gem/modulefiles 」

$\hookrightarrow$ /lustre-cray_gem_s/2.8.2_3.0.101_0.46.1_1.0502.8 」

$\hookrightarrow \quad 871-1.0502 .0 .44 .1: /$ opt/cray/gem/modulefiles/udre

$\hookrightarrow$ g/2.3.2-1.0502.10518.2.17.gem:/opt/cray/gem/modu」

$\hookrightarrow$ lefiles/ugni/6.0-1.0502.10863.8.28.gem:/opt/cray

$\hookrightarrow$ /gem/modulefiles/gni-headers/4.0-1.0502.10859.9. J

$\hookrightarrow$ 26.gem:/opt/cray/gem/modulefiles/dmapp/7.0.1-1.0

$\hookrightarrow$ 502.11080.8.74.gem:/opt/cray/gem/modulefiles/xpm 」

$\hookrightarrow$ em/0.1-2.0502.64982.7.26.gem:/opt/modulefiles/hs 」

$\hookrightarrow$ s- $11 \mathrm{~m} / 7.2 .0: / o p t / m o d u l e f i l e s / B a s e-o p t s / 1.0 .2-1.0$ ।

$\hookrightarrow$ 502.60680.2.4.gem:/opt/modulefiles/pgi/18.4.0:/o

$\hookrightarrow \mathrm{pt} / \mathrm{cray} / \mathrm{craype} / 2.5 .13 / \mathrm{modulefiles/craype-network」}$

$\hookrightarrow$-gemini:/opt/cray/craype/2.5.13/modulefiles/crayp」

$\hookrightarrow$ e-interlagos:/opt/cray/modulefiles/craype/2.5.13 」

$\hookrightarrow \quad:$ /opt/cray/modulefiles/cray-mpich/7.6.3:/opt/cra

$\hookrightarrow$ y/modulefiles/cray-libsci/16.11.1:/opt/cray/gem/ 」

$\hookrightarrow$ modulefiles/pmi/5.0.9-1.0000.10911.175.4.gem:/op ।

$\hookrightarrow$ t/cray/modulefiles/atp/2.1.1:/opt/cray/modulefil 」

$\hookrightarrow$ es/PrgEnv-pgi/5.2.82:/sw/xk6/modulefiles/lustred

$\hookrightarrow \mathrm{u} / 1.4: / \mathrm{sw} / \mathrm{xk} 6 / \mathrm{modulefiles} / \mathrm{xalt} / 0.7 .5: / \mathrm{sw} / \mathrm{xk} 6 / \mathrm{mod}$

$\hookrightarrow$ ulefiles/git/2.13.0:/sw/xk6/modulefiles/module_m」

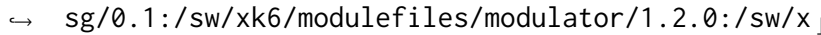

$\hookrightarrow$ k6/modulefiles/hsi/5.0.2.p1:/sw/xk6/modulefiles/

$\hookrightarrow$ DefApps:/opt/modulefiles/gcc/6.3.0:/opt/cray/mod」

$\hookrightarrow$ ulefiles/cudatoolkit/9.1.85_3.10-1.0502.df1cc54.」

$\hookrightarrow \quad 3.1$

PE_LIBSCI_DEFAULT_GENCOMPS_INTEL_mic_knl $=150$

PE_LIBSCI_DEFAULT_OMP_REQUIRES=

PE_MPICH_DEFAULT_GENCOMPS_CRAY $=86$

PE_PETSC_DEFAULT_GENCOMPILERS_GNU_sandybridge $=5.34 .9$

PE_TPSL_DEFAULT_GENCOMPILERS_INTEL_haswell $=16.0$

PE_LIBSCI_ACC_DEFAULT_NV_SUFFIX_nvidia $20=n v 20$ 
Duan, et al.

PE_LIBSCI_MODULE_NAME $=c r a y-l i b s c i / 16.11 .1$

PE_PETSC_DEFAULT_GENCOMPILERS_CRAY_skylake $=8.6$ PE_TPSL_DEFAULT_GENCOMPILERS_CRAY_interlagos $=8.6$ PE_TPSL_DEFAULT_GENCOMPILERS_GNU_mic_knl=5.1 PBS_NODENUM $=0$

LANG $=$ en_US.UTF-8

PE_LIBSCI_DEFAULT_GENCOMPILERS_PGI_x86_64=15.3

PE_LIBSCI_GENCOMPS_PGI_X86_64 $=153$

PE_TPSL_64_DEFAULT_GENCOMPS_GNU_x86_skylake $=61$

PE_INTEL_FIXED_PKGCONFIG_PATH=/opt/cray/mpt/7.6.3/gn」

$\hookrightarrow \quad$ i/mpich-intel/16.0/lib/pkgconfig

PGROUPD_LICENSE_FILE=/opt/pgi/license.dat

SYSTEM_USERDIR=/tmp/work/USER

MOABHOMEDIR=/opt/moab

PYTHONSTARTUP=/etc/pythonstart

MODULEPATH=/opt/cray/craype/2.5.13/modulefiles:/opt/ 」

$\hookrightarrow$ cray/gem/modulefiles:/opt/cray/modulefiles:/opt/」

$\hookrightarrow$ modulefiles:/sw/xk6/modulefiles:/sw/xk7/modulefi

$\hookrightarrow$ les:/lustre/atlas/scratch/USER/csc242/sw/titan/m

$\hookrightarrow$ odulefiles:/ccs/proj/env003/jyc/titan/sw/modulef

$\hookrightarrow$ iles:/lustre/atlas/world-shared/csc143/jyc/titan」

$\hookrightarrow$ /sw/modulefiles:/ccs/home/USER/modulefiles

PE_ACML_DEFAULT_VOLATILE_PKGCONFIG_PATH=/opt/acml/5. 」

$\hookrightarrow$ 3.1/@PE_ACML_DEFAULT_DIR@@PE_ACML_DEFAULT_TARGET 」

$\hookrightarrow$ @@PE_ACML_DEFAULT_DIR_OPENMP@@PE_ACML_DEFAULT_DI 」

$\hookrightarrow$ R_DEFAULT64@/lib/pkgconfig

PE_LIBSCI_GENCOMPILERS_CRAY_X86_64 $=8.3$

PE_MPICH_NV_LIBS_nvidia20=-1cudart

PE_MPICH_VOLATILE_PKGCONFIG_PATH=/opt/cray/mpt/7.6.3 」

$\hookrightarrow$ /gni/mpich-@PRGENV@@PE_MPICH_DIR_DEFAULT64@/@PE_」

$\hookrightarrow$ MPICH_GENCOMPSe/lib/pkgconfig

PBS_NUM_NODES $=1$

SDK_HOME $=/$ usr $/ \mathrm{lib} 64 / \mathrm{jvm} / \mathrm{java}$

LOADEDMODULES=modules $/ 3.2 \cdot 10.6:$ nodestat $/ 2.2-1.0502 .6$ 」

$\hookrightarrow$ 0539.1.31.gem:sdb/1.1-1.0502.63652.4.27.gem:alps」

$\hookrightarrow \quad / 5.2 .4-2.0502 .9950 .37 .1$.gem:lustre-cray_gem_s/2.」

$\hookrightarrow$ 8.2_3.0.101_0.46.1_1.0502.8871-1.0502.0.44.1:udr 」

$\hookrightarrow$ eg/2.3.2-1.0502.10518.2.17.gem:ugni/6.0-1.0502.1」

$\hookrightarrow$ 0863.8.28.gem:gni-headers/4.0-1.0502.10859.9.26.」

$\hookrightarrow$ gem:dmapp/7.0.1-1.0502.11080.8.74.gem:xpmem/0.1-」

$\hookrightarrow$ 2.0502.64982.7.26.gem:hss-11m/7.2.0:Base-opts/1.

$\hookrightarrow \quad 0.2-1.0502 .60680 .2 .4 . g e m: p g i / 18.4 .0:$ craype-netwo $_{\perp}$

$\hookrightarrow \quad$ rk-gemini:craype-interlagos:craype/2.5.13:cray-m 」

$\hookrightarrow$ pich/7.6.3:cray-libsci/16.11.1:pmi/5.0.9-1.0000.」

$\hookrightarrow$ 10911.175.4.gem:atp/2.1.1:PrgEnv-pgi/5.2.82:1ust

$\hookrightarrow$ redu/1.4:xalt/0.7.5:git/2.13.0:module_msg/0.1:mo」

$\hookrightarrow$ dulator/1.2.0:hsi/5.0.2.p1:DefApps:gcc/6.3.0:cud 」

$\hookrightarrow$ atoolkit/9.1.85_3.10-1.0502.df1cc54.3.1

SHMEM_ABORT_ON_ERROR=1

OLCF_GIT_ROOT=/autofs/nccs-svm1_sw/titan/.swci/0-log 」

$\hookrightarrow$ in/opt/spack/20170612/linux-suse_linux11-x86_64/」

$\hookrightarrow$ gcc-5.3.0/git-2.13.0-znpqlkovoclvlt5rwm3rkpk7d2m」

$\hookrightarrow \quad 56 \mathrm{ez} 2$

CRAY_PORTALS_USE_BLOCKING_POLL=no

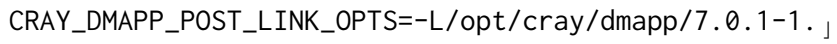

$\hookrightarrow \quad 0502.11080 .8 .74 . g e m / 1 i b 64$
PE_FFTW_DEFAULT_TARGET_ivybridge=ivybridge

PE_FFTW_DEFAULT_TARGET_share=share

PE_FFTW_DEFAULT_TARGET_x86_skylake=x86_skylake

PE_PKG_CONFIG_PATH=/opt/cray/cti $/ 1.0 .6 / \mathrm{lib} / \mathrm{pkgconfig}$ 」

$\hookrightarrow \quad:$ /opt/cray/cti/1.0.4/lib/pkgconfig:/opt/cray/cti 」

$\hookrightarrow / 1.0 .1 / \mathrm{lib} /$ pkgconfig

PE_TPSL_64_DEFAULT_GENCOMPS_GNU_interlagos $=5149$

PE_TPSL_DEFAULT_GENCOMPILERS_INTEL_mic_knl=16.0

PE_TPSL_DEFAULT_GENCOMPS_PGI_X86_64=153

PBS_0_SHELL=/bin/bash

PE_LIBSCI_OMP_REQUIRES_openmp=_mp

PE_PETSC_DEFAULT_GENCOMPILERS_GNU_skylake $=6.1$

PE_TPSL_DEFAULT_GENCOMPILERS_CRAY_x86_skylake $=8.6$

PE_TPSL_64_DEFAULT_GENCOMPS_CRAY_mic_knl $=86$

PE_TPSL_DEFAULT_GENCOMPILERS_INTEL_x86_64=16.0

CRAY_MPICH_DIR=/opt/cray/mpt/7.6.3/gni/mpich-pgi/15.3

PE_MPICH_CXX_PKGCONFIG_LIBS=mpichcxx

PBS_JOBID $=4642066$

PE_LIBSCI_ACC_DEFAULT_GENCOMPILERS_GNU_X86_64=4.9

PE_LIBSCI_DEFAULT_GENCOMPS_INTEL_X86_64=150

PE_MPICH_PKGCONFIG_VARIABLES=PE_MPICH_NV_LIBS_@accel 」

$\hookrightarrow$ erator@:PE_MPICH_ALTERNATE_LIBS_@multithreaded@: 」

$\hookrightarrow$ PE_MPICH_ALTERNATE_LIBS_@dpm@

ENVIRONMENT=BATCH

CRAY_PMI_POST_LINK_OPTS=-L/opt/cray/pmi / 5.0.9-1.0000 」

$\hookrightarrow \quad .10911 .175 .4$. gem/lib64

PE_HDF5_DEFAULT_FIXED_PRGENV=CRAY PGI INTEL

PE_TPSL_64_DEFAULT_GENCOMPILERS_CRAY_mic_knl=8.6

PE_TPSL_DEFAULT_GENCOMPILERS_INTEL_X86_skylake $=16.0$

PE_TPSL_DEFAULT_VOLATILE_PKGCONFIG_PATH=/opt/cray/tp 」

$\hookrightarrow$ sl/17.11.1/@PRGENV@/@PE_TPSL_DEFAULT_GENCOMPS@/@ 」

$\hookrightarrow$ PE_TPSL_DEFAULT_TARGET@/lib/pkgconfig

CRAY_MPICH2_VER=7.6.3

PE_MPICH_PKGCONFIG_LIBS=mpich

WORLDWORK=/lustre/atlas/world-shared

PE_GA_DEFAULT_GENCOMPILERS_GNU=5.1 4.9

PE_LIBSCI_ACC_DEFAULT_GENCOMPS_GNU_X86_64=49

PE_LIBSCI_VOLATILE_PKGCONFIG_PATH=/opt/cray/libsci $/ 1$ 」

$\hookrightarrow \quad 6.11 .1 /$ /@RGENVQ/@PE_LIBSCI_GENCOMPS@/@PE_LIBSCI _ 」

$\hookrightarrow$ TARGETQ/lib/pkgconfig

PE_MPICH_ALTERNATE_LIBS_mult $\mathrm{t}$ threaded=_mt

PE_NETCDF_DEFAULT_FIXED_PRGENV=CRAY PGI INTEL

PE_PARALLEL_NETCDF_DEFAULT_FIXED_PRGENV=CRAY PGI

$\hookrightarrow \quad$ INTEL

PE_TPSL_DEFAULT_GENCOMPS_PGI_interlagos $=153$

CUDATOOLKIT_HOME=/opt/nvidia/cudatoolkit9.1/9.1.85_3」

$\hookrightarrow \quad .10-1.0502 . \mathrm{df} 1 \mathrm{cc} 54.3 .1$

HOME $=/$ ccs/home/USER

SHLVL $=4$

JDK_HOME=/usr/lib64/jvm/java

QT_SYSTEM_DIR=/usr/share/desktop-data

CRAY_LIBSCI_VERSION $=16.11 .1$

PE_HDF5_PARALLEL_DEFAULT_VOLATILE_PRGENV=GNU

PE_MPICH_TARGET_VAR_nvidia35=-lcudart

PE_NETCDF_HDF5PARALLEL_DEFAULT_VOLATILE_PRGENV=GNU

PE_PKGCONFIG_PRODUCTS_DEFAULT=PE_PAPI 
PE_TPSL_64_DEFAULT_GENCOMPS_GNU_haswell=51 49

MEMBERWORK=/lustre/atlas/scratch/USER

OLCF_MODULATOR_SESSION=1554915651.4094. ti tan-batch3.

$\hookrightarrow \quad$ CCS.ornl.gov

OSTYPE=linux

LESS_ADVANCED_PREPROCESSOR $=$ no

PE_LIBSCI_GENCOMPILERS_PGI_X86_64 $=15.3$

PE_TPSL_DEFAULT_GENCOMPILERS_INTEL_interlagos $=16.0$

PGI_PATH=/opt/pgi/18.4.0

PE_ACML_DEFAULT_DIR_OPENMP=_mp

PE_LIBSCI_ACC_DEFAULT_NV_SUFFIX_nvidia $60=n v 60$

PE_MPICH_DEFAULT_VOLATILE_PKGCONFIG_PATH=/opt/cray $/ \mathrm{m}$ 」

$\hookrightarrow \mathrm{pt} / 7.6 .3 / \mathrm{gni} / \mathrm{mpich}$-@PRGENV@@PE_MPICH_DEFAULT_DIR 」

$\hookrightarrow$ _DEFAULT64@/@PE_MPICH_DEFAULT_GENCOMPS@/lib/pkgc 」

$\hookrightarrow$ onfig

PE_PETSC_DEFAULT_GENCOMPILERS_CRAY_interlagos $=8.6$

PE_TPSL_DEFAULT_VOLATILE_PRGENV=CRAY CRAY64 GNU GNU64

$\hookrightarrow \quad$ INTEL INTEL64 PGI PGI64

OLCF_MODULE_MSG $=/ \mathrm{sw} /$ sources/module_msg/0.1

PBS_O_HOST=titan-ext3.ccs.ornl.gov

XCURSOR_THEME $=$ crystalwhite

LS_OPTIONS $=-\mathrm{N}--$ color $=$ none $-\mathrm{T} 0$

CRAY_PMI_INCLUDE_OPTS=-I/opt/cray/pmi/5.0.9-1.0000.1 」

$\hookrightarrow$ 0911.175.4.gem/include

PE_TPSL_64_DEFAULT_GENCOMPS_CRAY_interlagos $=86$

PE_TPSL_DEFAULT_GENCOMPS_INTEL_sandybridge $=160$

CRAY_CUDATOOLKIT_INCLUDE_OPTS=-I/opt/nvidia/cudatool 」

$\hookrightarrow$ kit9.1/9.1.85_3.10-1.0502.df1cc54.3.1/include

$\hookrightarrow \quad$-I/opt/nvidia/cudatoolkit9.1/9.1.85_3.10-1.0502. 」

$\hookrightarrow$ df1cc54.3.1/extras/CUPTI/include

$\hookrightarrow \quad$-I/opt/nvidia/cudatoolkit9.1/9.1.85_3.10-1.0502.」

$\hookrightarrow$ df1cc54.3.1/extras/Debugger/include

CRAY_CUDATOOLKIT_DIR=/opt/nvidia/cudatoolkit9.1/9.1. 」 $\hookrightarrow$ 85_3.10-1.0502.df1cc54.3.1

WINDOWMANAGER=

PRGENVMODULES=PrgEnv-cray:PrgEnv-gnu:PrgEnv-intel:Pr 」

$\hookrightarrow$ gEnv-pathscale:PrgEnv-pgi

CRAY_LLM_DIR=/opt/cray $/ 1 \mathrm{~lm} / \mathrm{default}$

CRAYPE_NETWORK_TARGET=gemini

ATP_MRNET_COMM_PATH=/opt/cray/atp/2.1.1/libexec/atp_ 」

$\hookrightarrow$ mrnet_commnode_wrapper

PE_TPSL_DEFAULT_GENCOMPILERS_CRAY_haswell $=8.6$

PKG_CONFIG_PATH_DEFAULT=/opt/cray/papi/5.6.0.2/lib64」

$\hookrightarrow /$ pkgconfig

PE_MPICH_DIR_CRAY_DEFAULT64 $=64$

GCC_PATH=/opt/gcc/6.3.0

PBS_VNODENUM $=0$

PE_LIBSCI_GENCOMPILERS_INTEL_mic_knl $=15.0$

PE_PETSC_DEFAULT_GENCOMPILERS_GNU_haswell=5.3 4.9

PE_TPSL_64_DEFAULT_GENCOMPILERS_GNU_mic_knl $=5.1$

PE_TPSL_DEFAULT_GENCOMPILERS_GNU_interlagos $=5.14 .9$

PE_TPSL_DEFAULT_GENCOMPILERS_INTEL_sandybridge $=16.0$

PE_TPSL_DEFAULT_GENCOMPILERS_PGI_interlagos $=15.3$

LOGNAME $=$ USER

MACHTYPE $=x 86 \_64-$ suse-1inux

LESS $=-M-I$
G_FILENAME_ENCODING=@locale,UTF-8, ISO-8859-15, CP1252 CRAY_GNI_HEADERS_INCLUDE_OPTS=-I/opt/cray/gni-header 」 $\hookrightarrow s / 4.0-1.0502 .10859 .9 .26$. gem/include

PYTHONPATH=/sw/xk6/xalt/0.7.5/site:/sw/xk6/xalt/0.7. $\hookrightarrow$ 5/libexec:/opt/cray/sdb/1.1-1.0502.63652.4.27.ge

$\hookrightarrow \mathrm{m} / \mathrm{lib64/py:/ccs/home/USER/.} \mathrm{local/summit/anaconda} \mathrm{」}$ $\hookrightarrow$ 3/5.3.0/3.6/lib/python3.6/site-packages

CRAY_LIBSCI_PREFIX_DIR=/opt/cray/libsci/16.11.1/PGI/ $\hookrightarrow$ 15.3/X86_64

PE_HDF5_DEFAULT_GENCOMPS_GNU $=5149$

PE_MPICH_NV_LIBS =

PE_NETCDF_DEFAULT_REQUIRED_PRODUCTS=PE_HDF 5

PE_TPSL_64_DEFAULT_GENCOMPILERS_GNU_haswell=5.1 4.9

PE_TPSL_64_DEFAULT_GENCOMPILERS_INTEL_sandybridge $=16$ 」 $\hookrightarrow \quad .0$

PE_TPSL_DEFAULT_GENCOMPS_GNU_X86_64=51 49

PE_TRILINOS_DEFAULT_REQUIRED_PRODUCTS=PE_MPICH :PE_HD 」

$\hookrightarrow \quad$ F5_PARALLEL:PE_NETCDF_HDF5PARALLEL:PE_LIBSCI:PE_

$\hookrightarrow$ TPSL

CVS_RSH=sSh

DMAPP_ABORT_ON_ERROR $=1$

PE_LIBSCI_OMP_REQUIRES=

PE_MPICH_DEFAULT_GENCOMPILERS_CRAY $=8.6$

PE_TRILINOS_DEFAULT_GENCOMPS_GNU_X86_64=51 49

PE_MPICH_GENCOMPS_CRAY $=86$

PBS_QUEUE $=$ debug

XDG_DATA_DIRS=/usr/local/share:/usr/share:/etc/opt/k」

$\hookrightarrow$ de3/share:/opt/kde3/share

TOOLMODULES=apprentice : apprentice 2 : atp: chapel : cray-1

$\hookrightarrow$ gdb:cray-snplauncher:craypat:craypkg-gen:ddt:gdb」

$\hookrightarrow \quad$ :iobuf:papi:perftools:perftools-lite:stat: totalv $\rfloor$

$\hookrightarrow$ iew:xt-craypat:xt-lgdb:xt-papi:xt-totalview

PE_LIBSCI_ACC_DEFAULT_GENCOMPILERS_CRAY_x86_64=8.5

PE_LIBSCI_ACC_DEFAULT_NV_SUFFIX_nvidia $35=n v 35$

PE_LIBSCI_DEFAULT_REQUIRED_PRODUCTS=PE_MPICH

PE_MPICH_DEFAULT_FIXED_PRGENV=INTEL

PE_MPICH_DEFAULT_GENCOMPS_GNU $=5149$

PE_TPSL_64_DEFAULT_GENCOMPILERS_INTEL_interlagos $=16.0$ PE_TPSL_DEFAULT_GENCOMPILERS_CRAY_sandybridge $=8.6$

MODULESHOME $=/$ opt $/$ modules $/ 3.2 .10 .6$

PE_FFTW2_DEFAULT_REQUIRED_PRODUCTS=PE_MPICH

PE_GA_DEFAULT_FIXED_PRGENV=CRAY PGI INTEL

PE_LIBSCI_DEFAULT_VOLATILE_PKGCONFIG_PATH=/opt/cray/ 」

$\hookrightarrow \quad$ iibsci/16.11.1/@PRGENVQ/@PE_LIBSCI_DEFAULT_GENCO」

$\hookrightarrow$ MPS@/@PE_LIBSCI_DEFAULT_TARGET@/lib/pkgconfig

PE_TPSL_DEFAULT_GENCOMPILERS_GNU_sandybridge=5.1 4.9

PBS_O_MAIL=/var/mail/USER

PBS_MICFILE=/var/spool/torque/aux//4642066mic

LESSOPEN=lessopen. sh \%s 
PKG_CONFIG_PATH=/opt/nvidia/cudatoolkit9.1/9.1.85_3.」 $\hookrightarrow$ 10-1.0502.df1cc54.3.1/1 ib64/pkgconfig:/opt/cray/」 $\hookrightarrow \mathrm{rca} / 1.0 .0-2.0502 .60530 .1 .63 . \mathrm{gem} / \mathrm{lib64/pkgconfig:}$ $\hookrightarrow$ /opt/cray/pmi/5.0.9-1.0000.10911.175.4.gem/lib64」 $\hookrightarrow$ /pkgconfig:/opt/cray/craype/2.5.13/pkg-config:/o」 $\hookrightarrow \mathrm{pt} / \mathrm{cray} /$ iobuf/2.0.8/lib/pkgconfig:/opt/cray/fftw $\hookrightarrow$ /2.1.5.8/lib/pkgconfig:/opt/cray/xpmem/0.1-2.050 $\hookrightarrow \quad 2.64982 .7 .26$.gem/lib64/pkgconfig:/opt/cray/dmapp $\hookrightarrow$ /7.0.1-1.0502.11080.8.74.gem/lib64/pkgconfig:/op」 $\hookrightarrow \quad t / c r a y / g n i-h e a d e r s / 4.0-1.0502 .10859 .9 .26 . g e m / 1 i b 」$ $\hookrightarrow$ 64/pkgconfig:/opt/cray/ugni/6.0-1.0502.10863.8.2 $\hookrightarrow$ 8.gem/lib64/pkgconfig:/opt/cray/udreg/2.3.2-1.05」 $\hookrightarrow$ 02.10518.2.17.gem/lib64/pkgconfig:/opt/cray/alps」 $\hookrightarrow \quad / 5.2 .4-2.0502 .9950 .37 .1 . \mathrm{gem} / \mathrm{lib64/pkgconfig:/opt}$ $\hookrightarrow$ /cray/atp/2.1.1/lib/pkgconfig:/ccs/home/USER/sum 」 $\hookrightarrow \mathrm{mit} / \mathrm{sw} / \mathrm{libfabric/lib/pkgconfig}$

PE_MPICH_NV_LIBS_nvidia35=-lcudart

PE_PETSC_DEFAULT_VOLATILE_PKGCONFIG_PATH=/opt/cray/p 」

$\hookrightarrow$ etsc/3.7.6.3/complex/@PRGENVQ/QPE_PETSC_DEFAULT_」 $\hookrightarrow \quad$ GENCOMPS@/@PE_PETSC_DEFAULT_TARGET@/lib/pkgconfig PELOCAL_PRGENV=true

LIBSCI_BASE_DIR=/opt/cray/libsci/16.11.1

PE_TPSL_64_DEFAULT_GENCOMPS_INTEL_x86_64=160

INFOPATH=/opt/gcc/6.3.0/snos/share/info:/usr/local/i $\hookrightarrow \mathrm{nfo} /$ usr/share/info:/usr/info

LIBSCI_VERSION=16.11.1

PE_LIBSCI_DEFAULT_PKGCONFIG_VARIABLES=PE_LIBSCI_DEFA 」 $\hookrightarrow \quad$ ULT_OMP_REQUIRES_@openmp@

PE_MPICH_NV_LIBS_nvidia60=-lcudart

PE_TPSL_64_DEFAULT_GENCOMPS_GNU_sandybridge $=5149$

PE_TPSL_DEFAULT_GENCOMPS_INTEL_mic_knl=160

PBS_NP=16

CRAY_ALPS_INCLUDE_OPTS=-I/opt/cray/alps/5.2.4-2.0502」 $\hookrightarrow \quad .9950 .37 .1$.gem/include

CRAY_PRE_COMPILE_OPTS=-hnetwork=gemini

PE_FFTW_DEFAULT_TARGET_broadwell=broadwell

PE_LIBSCI_GENCOMPILERS_INTEL_x86_64 $=15.0$

PE_PGI_DEFAULT_FIXED_PKGCONFIG_PATH=/opt/cray/parall」

$\hookrightarrow$ el-netcdf/1.8.1.3/PGI/15.3/lib/pkgconfig:/opt/cr」

$\hookrightarrow$ ay/netcdf-hdf5parallel/4.4.1.1.3/PGI/15.3/lib/pk」

$\hookrightarrow$ gconfig:/opt/cray/netcdf/4.4.1.1.3/PGI/15.3/1ib/」

$\hookrightarrow$ pkgconfig:/opt/cray/hdf5-parallel/1.10.0.3/PGI/1」

$\hookrightarrow$ 5.3/lib/pkgconfig:/opt/cray/hdf5/1.10.0.3/PGI/15」

$\hookrightarrow$.3/lib/pkgconfig:/opt/cray/ga/5.3.0.7/PGI/15.3/1」

$\hookrightarrow \quad \mathrm{ib} /$ pkgconfig

PE_TPSL_64_DEFAULT_GENCOMPILERS_GNU_X86_64=5.1 4.9

CRAY_CPU_TARGET=interlagos

CMAKE_PREFIX_PATH=/autofs/nccs-svm1_sw/titan/.swci/0 」

$\hookrightarrow-$ - login/opt/spack/20170612/linux-suse_linux11-x86_」

$\hookrightarrow$ 64/gcc-5.3.0/git-2.13.0-znpqlkovoclvlt5rwm3rkpk7」

$\hookrightarrow \mathrm{d} 2 \mathrm{~m} 56 \mathrm{ez} 2 /$

XTPE_LINK_TYPE=dynamic

PBS_0_SERVER=ti tan-moab.ccs.ornl.gov

PBS_NUM_PPN $=16$

CRAY_UGNI_INCLUDE_OPTS=-I/opt/cray/ugni/6.0-1.0502.1 」

$\hookrightarrow \quad 0863.8 .28$.gem/include
CRAY_XPMEM_INCLUDE_OPTS=-I/opt/cray/xpmem/0.1-2.0502 」 $\hookrightarrow \quad .64982 .7 .26$.gem/include

PE_LIBSCI_DEFAULT_GENCOMPS_CRAY_mic_knl=85

PE_LIBSCI_REQUIRED_PRODUCTS=PE_MPICH

PE_MPICH_DEFAULT_GENCOMPILERS_PGI $=15.3$

PE_PAPI_DEFAULT_ACCELL_FAMILY_LIBS=

PE_TPSL_64_DEFAULT_GENCOMPS_CRAY_X86_64 $=86$

PE_MPICH_GENCOMPS_PGI $=153$

PE_LIBSCI_DEFAULT_GENCOMPILERS_GNU_mic_knl=5.1

PE_LIBSCI_DEFAULT_GENCOMPILERS_GNU_X86_64=6.1 5.14 .9

PE_LIBSCI_GENCOMPS_GNU_mic_knl=51

PE_LIBSCI_GENCOMPS_GNU_X86_64=61 5149

PE_TPSL_DEFAULT_GENCOMPS_INTEL_haswell $=160$

LESSCLOSE $=$ lessclose.sh \%s \%

ATP_HOME=/opt/cray/atp/2.1.1

PE_FFTW_DEFAULT_TARGET_x86_64=x86_64

PE_PETSC_DEFAULT_GENCOMPILERS_INTEL_x86_64=16.0

PBS_NODEFILE=/var/spool/torque/aux//4642066

G_BROKEN_FILENAMES $=1$

CRAY_LD_LIBRARY_PATH=/opt/nvidia/cudatoolkit9.1/9.1. 」 $\hookrightarrow$ 85_3.10-1.0502.df1cc54.3.1/1ib64:/opt/nvidia/cud」

$\hookrightarrow$ atoolkit9.1/9.1.85_3.10-1.0502.df1cc54.3.1/extra 」

$\hookrightarrow$ s/CUPTI/lib64:/opt/cray/pmi/5.0.9-1.0000.10911.1」

$\hookrightarrow$ 75.4.gem/lib64:/opt/cray/libsci/16.11.1/PGI/15.3」

$\hookrightarrow$ /x86_64/lib:/opt/cray/mpt/7.6.3/gni/mpich-pgi/15」

$\hookrightarrow$.3/lib:/opt/cray/xpmem/0.1-2.0502.64982.7.26.gem」

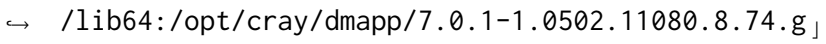

$\hookrightarrow$ em/lib64:/opt/cray/ugni/6.0-1.0502.10863.8.28.ge J

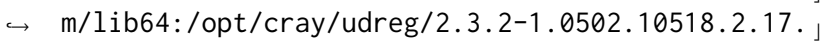

$\hookrightarrow$ gem/lib64:/opt/cray/alps/5.2.4-2.0502.9950.37.1.」

$\hookrightarrow$ gem/lib64

CRAYOS_VERSION $=5.2 .82$

PE_FFTW_DEFAULT_TARGET_haswell=haswell

PE_GA_DEFAULT_GENCOMPS_GNU $=5149$

PE_GA_DEFAULT_VOLATILE_PKGCONFIG_PATH=/opt/cray/ga/5 」

$\hookrightarrow \quad$.3.0.7/@PRGENV@/@PE_GA_DEFAULT_GENCOMPS@/lib/pkg 」

$\hookrightarrow$ config

PE_INTEL_DEFAULT_FIXED_PKGCONFIG_PATH=/opt/cray/para 」

$\hookrightarrow$ llel-netcdf/1.8.1.3/INTEL/16.0/lib/pkgconfig:/op」

$\hookrightarrow \quad t / c r a y /$ netcdf-hdf5parallel/4.4.1.1.3/INTEL/16.0/」

$\hookrightarrow$ lib/pkgconfig:/opt/cray/netcdf/4.4.1.1.3/INTEL/1」

$\hookrightarrow$ 6.0/lib/pkgconfig:/opt/cray/mpt/7.6.3/gni/mpich-」

$\hookrightarrow$ intel/16.0/lib/pkgconfig:/opt/cray/hdf5-parallel」

$\hookrightarrow \quad / 1.10 .0 .3 /$ INTEL/16.0/lib/pkgconfig:/opt/cray/hdf J

$\hookrightarrow \quad 5 / 1.10 .0 .3 /$ INTEL/16.0/lib/pkgconfig:/opt/cray/ga 」

$\hookrightarrow \quad / 5.3 .0 .7 /$ INTEL/15.0/lib/pkgconfig

PE_PAPI_DEFAULT_ACCEL_LIBS=

PE_PETSC_DEFAULT_GENCOMPILERS_GNU_interlagos=5.3 4.9 PE_PETSC_DEFAULT_GENCOMPILERS_INTEL_haswell $=16.0$

PE_SMA_DEFAULT_DIR_PGI_DEFAULT T4 $=64$

PE_TPSL_64_DEFAULT_GENCOMPILERS_INTEL_x86_skylake $=16$ 」 $\hookrightarrow \quad .0$ 
PBS_0_PATH=/opt/nvidia/cudatoolkit9.1/9.1.85_3.10-1.」 $\hookrightarrow$ 0502.df1cc54.3.1/bin:/opt/nvidia/cudatoolkit9.1/」 $\hookrightarrow 9.1 .85 \_3.10-1.0502$.df1cc54.3.1/libnvvp:/opt/gcc/」 $\hookrightarrow$ 6.3.0/bin:/ccs/home/USER/codar/spack/bin:/sw/xk6」

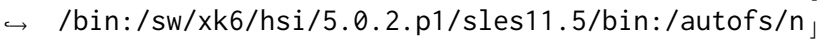
$\hookrightarrow$ ccs-svm1_sw/titan/.swci/0-login/opt/spack/201706」

$\hookrightarrow$ 12/linux-suse_linux11-x86_64/gcc-5.3.0/git-2.13.」 $\hookrightarrow \quad 0$-znpqlkovoclvlt5rwm3rkpk7d2m56ez2/bin:/sw/xk6/x 」 $\hookrightarrow$ alt/0.7.5/bin:/sw/xk6/lustredu/1.4/sles11.3_gnu4」 $\hookrightarrow$.8.2/bin:/opt/cray/mpt/7.6.3/gni/bin:/opt/cray/r」 $\hookrightarrow \mathrm{ca} / 1.0 .0-2.0502 .60530 .1 .63 . \mathrm{gem} / \mathrm{bin}: / o p t / \mathrm{cray} / \mathrm{alp}$ 」 $\hookrightarrow \mathrm{s} / 5.2 .4-2.0502 .9774 .31 .12 . \mathrm{gem} / \mathrm{sbin}$ /opt/cray/dvs 」 $\hookrightarrow$ /2.5_0.9.0-1.0502.2188.1.113.gem/bin:/opt/cray/x」 $\hookrightarrow$ pmem/0.1-2.0502.64982.5.3.gem/bin:/opt/cray/ugni $\hookrightarrow \quad$ /6.0-1.0502.10863.8.28.gem/bin:/opt/cray/udreg/2 」 $\hookrightarrow \quad .3 .2-1.0502 .10518 .2 .17$.gem/bin:/opt/cray/craype/」 $\hookrightarrow$ 2.5.13/bin:/opt/pgi/18.4.0/linux86-64/18.4/bin:/」 $\hookrightarrow$ opt/cray/eslogin/eswrap/1.3.3-1.020200.1280.0/bi 」

$\hookrightarrow \mathrm{n}$ :/usr/bin:/usr/sbin:/opt/moab/bin:/ccs/home/USE 」

$\hookrightarrow$ R/bin:/usr/local/bin:/usr/bin:/bin:/usr/bin/X11:」

$\hookrightarrow$ /usr/X11R6/bin:/usr/games:/opt/bin:/usr/lib/mit/」

$\hookrightarrow$ bin:/usr/lib/mit/sbin:/sbin:/usr/sbin:/usr/lib/q」

$\hookrightarrow$ t3/bin:/opt/cray/bin:/opt/bin:/opt/public/bin:/o」

$\hookrightarrow$ pt/bin:/opt/public/bin:/cm/local/apps/environmen」

$\hookrightarrow$ t-modules/3.2.10/bin

COLORTERM $=1$

JAVA_ROOT=/usr/lib64/jvm/java

PE_MPICH_DEFAULT_DIR_CRAY_DEFAULT $64=64$

PE_PETSC_DEFAULT_GENCOMPS_CRAY_haswell $=86$

PE_PETSC_DEFAULT_GENCOMPS_GNU_X86_64 $=5349$

PE_PETSC_DEFAULT_GENCOMPS_INTEL_x86_64=160

PE_TPSL_64_DEFAULT_GENCOMPS_PGI_interlagos $=153$

BASH_FUNC_module ()$=()\{$ eval

$\hookrightarrow$ '/opt/modules/3.2.10.6/bin/modulecmd bash $\$ *^{\prime}$

\}

+ 1sb_release -a

LSB Version:

core-2.0-noarch:core-3.2-noarch: ।

$\hookrightarrow$ core-4.0-noarch:core-2.0-x86_64:core-3.2-x86_64:」

$\hookrightarrow$ core-4.0-x86_64:desktop-4.0-amd64:desktop-4.0-no」

$\hookrightarrow$ arch:graphics-2.0-amd64:graphics-2.0-noarch:grap」

$\hookrightarrow$ hics-3.2-amd64:graphics-3.2-noarch:graphics-4.0-」

$\hookrightarrow$ amd64:graphics-4.0-noarch

Distributor ID:

SUSE LINUX

Description:

SUSE Linux Enterprise Server 11

$\hookrightarrow \quad\left(x 86 \_64\right)$

Release:

Codename:

11

+ uname $-a$

Linux nid03772 3.0.101-0.46.1_1.0502.8871-cray_gem_c

$\hookrightarrow \quad \# 1$ SMP Mon Feb 4 22:33:39 UTC 2019 x86_64 x86_64

$\hookrightarrow \quad x 86 \_64$ GNU/Linux

+ lscpu

Architecture:

x86_64

CPU op-mode(s):

Byte Order:

CPU(s): 32-bit, 64-bit

16
Little Endian

\begin{tabular}{|c|c|c|}
\hline \multirow{4}{*}{\multicolumn{2}{|c|}{$\begin{array}{l}\text { On-line CPU(s) list: } \\
\text { Thread(s) per core: } \\
\text { Core(s) per socket: } \\
\text { Socket(s): }\end{array}$}} & $0-15$ \\
\hline & & 2 \\
\hline & & 8 \\
\hline & & 1 \\
\hline \multirow{2}{*}{\multicolumn{2}{|c|}{$\begin{array}{l}\text { NUMA node }(s): \\
\text { Vendor ID: }\end{array}$}} & 2 \\
\hline & & AuthenticAMD \\
\hline \multicolumn{2}{|l|}{ CPU family: } & 21 \\
\hline \multicolumn{2}{|l|}{ Model: } & 1 \\
\hline \multicolumn{2}{|l|}{ Stepping: } & 2 \\
\hline \multicolumn{2}{|l|}{ CPU MHz: } & 2200.000 \\
\hline \multicolumn{2}{|l|}{ BogoMIPS: } & 4400.00 \\
\hline \multicolumn{2}{|l|}{ Virtualization: } & AMD-V \\
\hline \multicolumn{2}{|l|}{ L1d cache: } & $16 K$ \\
\hline \multicolumn{2}{|l|}{ L1i cache: } & $64 \mathrm{~K}$ \\
\hline \multicolumn{2}{|l|}{ L2 cache: } & $2048 \mathrm{~K}$ \\
\hline \multicolumn{2}{|c|}{ L3 cache: } & $6144 \mathrm{~K}$ \\
\hline \multicolumn{2}{|c|}{ NUMA node0 $\operatorname{CPU}(\mathrm{s})$ : } & $\partial-7$ \\
\hline \multicolumn{2}{|c|}{$\begin{array}{l}\text { NUMA node1 CPU(s): } \\
+ \text { cat /proc/meminfo }\end{array}$} & $8-15$ \\
\hline \multicolumn{2}{|c|}{ MemTotal: $\quad 33083668$} & $8 \mathrm{kB}$ \\
\hline \multicolumn{2}{|l|}{ MemFree: } & $\mathrm{kB}$ \\
\hline Buffers: & & $\mathrm{kB}$ \\
\hline Cached: & 60484 & $4 \mathrm{kB}$ \\
\hline SwapCached: & & $\mathrm{kB}$ \\
\hline Active: & 25556 & $6 \mathrm{kB}$ \\
\hline Inactive: & 319636 & $6 \mathrm{kB}$ \\
\hline Active(anon): & 22032 & $2 \mathrm{kB}$ \\
\hline Inactive(anon): & 700 & $\mathrm{kB}$ \\
\hline Active(file): & 3524 & $4 \mathrm{kB}$ \\
\hline Inactive(file): & 318936 & $6 \mathrm{kB}$ \\
\hline Unevictable: & 68620 & $\mathrm{kB}$ \\
\hline Mlocked: & 21128 & $8 \mathrm{kB}$ \\
\hline SwapTotal: & & $\mathrm{kB}$ \\
\hline SwapFree: & & $\mathrm{kB}$ \\
\hline Dirty: & & $\mathrm{kB}$ \\
\hline Writeback: & & $\mathrm{kB}$ \\
\hline AnonPages: & 40368 & $8 \mathrm{kB}$ \\
\hline Mapped: & 5972 & $2 \mathrm{kB}$ \\
\hline Shmem: & 624 & $4 \mathrm{kB}$ \\
\hline Slab: & 732992 & $2 \mathrm{kB}$ \\
\hline SReclaimable: & 6024 & $4 \mathrm{kB}$ \\
\hline SUnreclaim: & 726968 & $8 \mathrm{kB}$ \\
\hline KernelStack: & 2160 & $\mathrm{kB}$ \\
\hline PageTables: & 1012 & $2 \mathrm{kB}$ \\
\hline NFS_Unstable: & & $\mathrm{kB}$ \\
\hline Bounce: & & $\mathrm{kB}$ \\
\hline WritebackTmp: & & $\mathrm{kB}$ \\
\hline CommitLimit: & 16541832 & $2 \mathrm{kB}$ \\
\hline Committed_AS: & 74408 & $8 \mathrm{kB}$ \\
\hline VmallocTotal: & 34359738 & $8367 \mathrm{kB}$ \\
\hline VmallocUsed: & 3916960 & $\mathrm{kB}$ \\
\hline VmallocChunk: & 34354632 & $2735 \mathrm{kB}$ \\
\hline HugePages_Total: & $: \quad 0$ & $\partial$ \\
\hline HugePages_Free: & 0 & $\partial$ \\
\hline HugePages_Rsvd: & 0 & $\partial$ \\
\hline HugePages_Surp: & 0 & $\partial$ \\
\hline Hugepagesize: & 2048 & $8 \mathrm{kB}$ \\
\hline
\end{tabular}




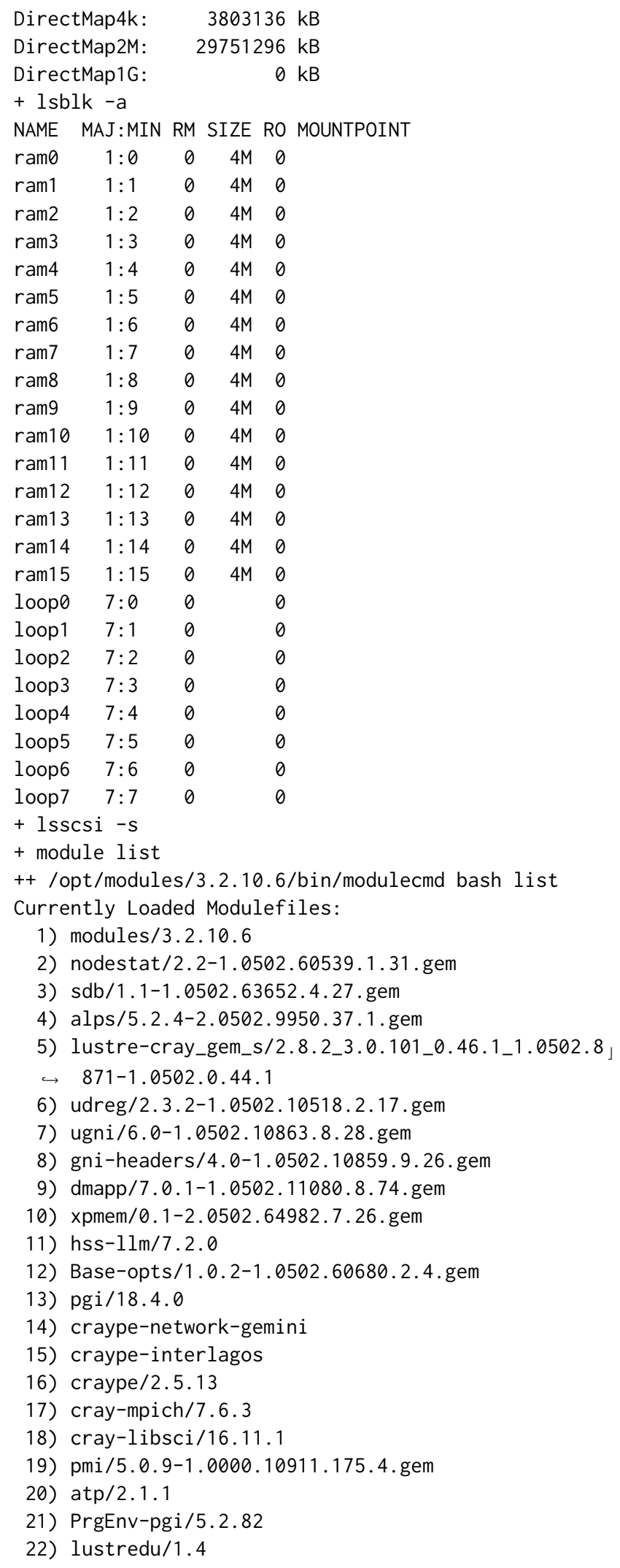

9) dmapp/7.0.1-1.0502.11080.8.74.gem

10) $x$ pmem $/ 0.1-2.0502 .64982 .7 .26 . \mathrm{gem}$

11) $\mathrm{hss}-1 \mathrm{~lm} / 7.2 .0$

12) Base-opts/1.0.2-1.0502.60680.2.4.gem

13) $\mathrm{pgi} / 18.4 .0$

14) craype-network-gemini

15) craype-interlagos

16) craype/2.5.13

17) cray-mpich/7.6.3

18) cray-libsci/16.11.1

19) $\mathrm{pmi} / 5.0 .9-1.0000 .10911 .175 .4 . \mathrm{gem}$

20) $a t p / 2.1 .1$

21) PrgEnv-pgi/5.2.82

22) lustredu/1.4

23) $x a l t / 0.7 .5$

24) git/2.13.0

25) module_msg/0.1

26) modulator $/ 1.2 .0$

27) hsi/5.0.2.p1

28) DefApps

29) $\mathrm{gcc} / 6.3 .0$

30) cudatoolkit/9.1.85_3.10-1.0502.df1cc54.3.1

+ eval 